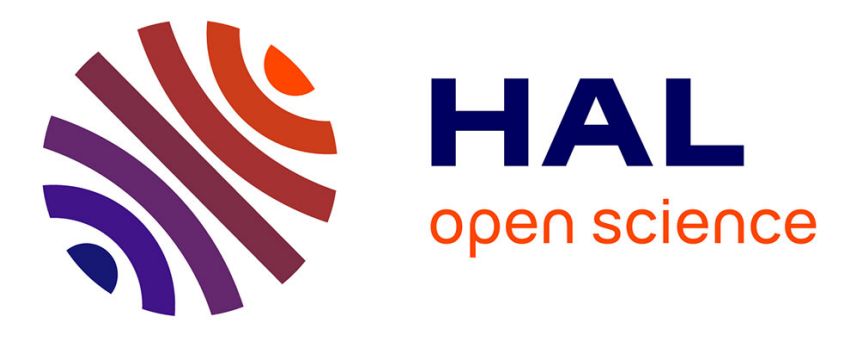

\title{
Multimodal wave propagation in smart composite structures with shunted piezoelectric patches
}

Tianli Huang, Mohamed Ichchou, Olivier Bareille, Manuel Collet, Morvan

Ouisse

\section{- To cite this version:}

Tianli Huang, Mohamed Ichchou, Olivier Bareille, Manuel Collet, Morvan Ouisse. Multimodal wave propagation in smart composite structures with shunted piezoelectric patches. Journal of Intelligent Material Systems and Structures, 2013, 24, pp.1155-1175. 10.1177/1045389X13480571 . hal00993369

\section{HAL Id: hal-00993369 \\ https://hal.science/hal-00993369}

Submitted on 20 May 2014

HAL is a multi-disciplinary open access archive for the deposit and dissemination of scientific research documents, whether they are published or not. The documents may come from teaching and research institutions in France or abroad, or from public or private research centers.
L'archive ouverte pluridisciplinaire HAL, est destinée au dépôt et à la diffusion de documents scientifiques de niveau recherche, publiés ou non, émanant des établissements d'enseignement et de recherche français ou étrangers, des laboratoires publics ou privés. 
This document is the author's final manuscript of

T.L. Huang, M.N. Ichchou, O. Bareille, M. Collet \& M. Ouisse: Multi-modal wave propagation in smart composite structures with shunted piezoelectric patches. Journal of Intelligent Material Systems and Structures, 2013.

This paper has been published by SAGE Journals and can be found at http://dx.doi.org/10.1177/1045389X13480571 


\title{
Multi-modal wave propagation in smart composite structures with shunted piezoelectric patches
}

\author{
T.L. Huang ${ }^{1}$, M.N. Ichchou ${ }^{1, *}$, O. Bareille ${ }^{1}$, M. Collet $^{2}$ and M. Ouisse ${ }^{2}$
}

${ }^{1}$ LTDS UMR5513 Ecole Centrale de Lyon, 36 Avenue Guy de Collongue, 69130 Ecully, France

${ }^{2}$ FEMTO-ST, Department of Applied Mechanics, UMR6174, 24 Chemin de l'Epitaphe, 25000 Besançon, France

*Corresponding author. E-mail: mohamed.ichchou@ec-lyon.fr

\begin{abstract}
Wave propagation in composite structures with shunted piezoelectric patches is investigated in this work. The Wave Finite Element(WFE) approach is firstly developed as a prediction tool for wave propagation characteristics like dispersion curves in composite structures, and subsequently extended to consider shunted piezoelectric elements through the Diffusion Matrix Model(DMM). A three-layered composite beam equipped with a pair of Resistor-Inductor(R-L) shunted piezoelectric patches is modeled and analyzed carefully with these numerical techniques. Reflection and transmission coefficients of propagating waves in this smart composite structure are calculated, and the performance of shunted piezoelectric patches on the control of wave propagation
\end{abstract}


is investigated numerically with the DMM. Another finite element formulation, named Modified Wave Finite Element(MWFE) method, which is dedicated to the analysis of wave propagation in multi-layered composite structures, is proposed and developed for considering piezoelectric elements in the structures. It is a dynamic substructuring technique that allows the dynamics of a typical layer cross-section to be projected on a reduced local wave mode basis with appropriate dimension. Results issued from this method are compared to those issued from the classical WFE and DMM formulations to demonstrate the pertinence of the modelings.

Keywords: Composite structure, propagation, energy diffusion, semi-active control, piezoelectric shunt.

\section{INTRODUCTION}

Multi-layered systems are widely used in many engineering domains, offering structural designers plenty of attractive features like high specific stiffness, good buckling resistance, formability into complex shapes, easy reparability, etc. Understanding, predicting and tailoring their vibratory behavior has always been an important issue in vibroacoustics. With the development of the finite element method (FEM), the analysis of the dynamic behavior of such structures is becoming more and more convenient, as this method possessed 
the advantages of widespread use in engineering domain and the capability of treating complex geometry. However, problems arise when large size models are treated, as the computational cost of the resolution becomes unacceptable, especially for time response calculations. As an alternative, the dynamic analysis of multi-layered systems based on the numerical descriptions of waves traveling in these structures can be applied, as it provides a low cost and efficient way to capture the dynamic behavior with relatively small models. This technique appears especially well suited for the short wavelength domain as the dynamics of systems can be accurately described by highly convergent reduced bases containing essential wave motions.

One of the two main objectives of the paper lies in the study of low-frequency (LF) and mid-frequency (MF) wave propagation in slender multi-layered elastic systems. Such systems exhibit large scale behavior associated with uniform cross-sections where are confined high regular "rigid body" and elastic modes, and fine scale behavior associated with low regular propagating components (Mencik and Ichchou, 2008). The frequency ranges can be defined as follows: the LF range refers to the frequency domain for which a typical cross-section contains a small number of elastic modes, e.g. the related "cross-section" modal density is small, while the MF range corresponds to an intermediate frequency domain between the LF range and the high-frequency (HF) range for which the cross-section modal density exhibits large variations (Ohayon and Soize, 1998). The Wave Finite Element (WFE) method (Mencik and Ichchou, 2008, 2005, 2007; Ichchou et al., 2007), which 
is based on the classic finite element description of a typical cell extracted from a given global system, is developed as an appropriate method for predicting the propagation in waveguides such as beams (Mace et al., 2005; Duhamel et al., 2006) and plates (Mace and Manconi, 2008; Collet et al., 2011) in a wide frequency range. Nevertheless, the WFE method suffers from a number of numerical problems especially when multi-layered structures are concerned. In order to address this issue, Mencik and Ichchou (2008) developed a substructuring technique named modified wave finite element (MWFE) for analyzing wave propagation in multi-layered systems, allowing the standard wave motions of multi-layered systems to be correctly captured. This technique will be applied in this paper to study the wave propagation in slender multi-layered elastic beams so as to achieve the first objective of this paper.

The second objective of this paper lies in the control of wave propagation in multi-layered systems. A recent revolution in the field of integrated microelectromechanical systems (MEMS) offers new opportunities for smart structure design. Smart composite structures is able to be created via the mechanical integration of active smart materials, electronics, chip sets and power supply systems (Collet et al., 2003; Meyer et al., 2007). The material's intrinsic passive mechanical behavior can be controlled through electromechanical transducers in order to attain new desired functionalities (Collet et al., 2009). Among the control configurations found in published work, a well-known technique is the piezoelectric damping using external resistor- 
inductor shunt circuit (Collet et al., 2009; Hagood and Flotow, 1991; Thorp et al., 2001; Nguyen and Pietrzko, 2006; Becker et al., 2006; Spadoni et al., 2009; Casadei et al., 2010). This semi-active configuration has the advantage of guaranteeing stability, and can be obtained by bonding piezoelectric elements onto a structure and connecting the electrodes to the external shunt circuit. Due to straining of the host structure, and through the direct piezoelectric effect, a portion of the mechanical energy is converted into electrical energy and subsequently be dissipated by Joule heating via the connected resistor. The $R-L$ shunt circuit on piezoelectric patches can be regarded as light oscillators instead of heavy mass-spring structures. By varying the inductance $L$ in the shunt circuit, the tuning frequency can be adjusted to desired frequency band. Recently, Spadoni et al. (2009) and Casadei et al. (2010) have studied the control of wave propagation in plates with periodic arrays of shunted piezoelectric patches. Efforts have been dedicated firstly to developing the finite element formulation of shunted piezoelectric elements, then to characterizing the dispersion relation of waves propagating over the surface of plate structures and the band gaps in the frequency domain. An experimental investigation was carried out in the work of Casadei et al. (2010) to test the performance of shunted piezoelectric patches via the forced response of the structure. Wang et al. (2011) have realized the same analysis on a beam with periodic shunted piezoelectric patches through an analytical model and corresponding experimental tests. Collet et al. (2009) provided a full finite element description of a beam with periodic shunted 
piezoelectric patches via the WFE method, but emphasis was placed on the optimization of shunt impedance. Numerical tools dedicated to the analysis of energy diffusion properties for multi-layered structures with shunted piezoelectric elements need to be properly developed in order to achieve the second objective of this paper.

This paper is organized as follows: in Section 2, a brief outline of the WFE approach is provided, as well as the DMM for the prediction of energy diffusion of wave modes propagating in the system(Subsection 2.1). The MWFE formulation is subsequently presented in Subsection 2.2 for the modeling of a multi-layered beam. Then the multi-layered beam with $R-L$ shunted piezoelectric patches can be modeled with the piezoelectric finite elemen$\mathrm{t}$ formulations given in Subsection 2.3. All these numerical techniques are applied in Section 3, where a three-layered beam is investigated with WFE and MWFE respectively. Results issued from these two approaches are compared. Parametric studies are also performed to investigate the influence of the dimension of mode bases on the prediction of wave propagation and diffusion characteristics. The performance of the shunted piezoelectric patches on the control of flexural wave mode in the multi-layered beam is tested. Concluding remarks and perspectives of this work are presented in Section 4. 


\section{NUMERICAL TOOLS FOR SMART COM- POSITE STRUCTURES WITH SHUNT- ED PIEZOELECTRIC ELEMENTS}

In this section, firstly the WFE formulation and the DMM are presented. After that, the MWFE formulation for multi-layered systems is given. Two different modelings for the global wave mode construction are described. Then the numerical models of a multi-layered beam with shunted piezoelectric elements are described. The finite element diffusion model proposed in the work of Mencik and Ichchou $(2008,2005)$ was extended to consider piezo-

electric elements. All these techniques enable the calculation of parameters like reflection and transmission coefficients so as to investigate the influence of the shunted piezoelectric patches on the propagation of wave modes in the multi-layered beam. The formulations developed are general and can be employed for all types of slender multi-layered structures with shunted piezoelectric patches.

\subsection{Wave propagation and diffusion in structures through finite elements}

The WFE method was employed to study the energy diffusion problem (Mencik and Ichchou, 2005; Ichchou et al., 2009; Huang et al., 2012a,b). The work of Mencik and Ichchou (2005) provides a detailed description of the dynamical 
behavior of a slender structure, as illustrated in Figure 1, which is composed, along a specific direction (say $X$-axis), of $N$ identical substructures. Note that this general description can be applied to homogeneous systems whose cross-sections are constant. The dynamic of the global system is formulated from the description of the waves propagating along the $X$-axis.

\section{Figure 1}

Let us consider a finite element model of a given substructure $k(k \in\{1, \ldots, N\})$ belonging to the waveguide (cf. Figure 1). The left and right boundaries of the discretized substructure are assumed to contain $n$ degrees of freedom (DOFs). Displacements $\mathbf{q}$ and forces $\mathbf{F}$ which are applied on these boundaries are denoted by $\left(\mathbf{q}_{\mathrm{L}}, \mathbf{q}_{\mathrm{R}}\right)$ and $\left(\mathbf{F}_{\mathrm{L}}, \mathbf{F}_{\mathrm{R}}\right)$, respectively. It is assumed

that the kinematic quantities are represented through state vectors $\mathbf{u}_{\mathrm{L}}^{(k)}=$ $\left(\left(\mathbf{q}_{\mathrm{L}}^{(k)}\right)^{\mathrm{T}}\left(-\mathbf{F}_{\mathrm{L}}^{(k)}\right)^{\mathrm{T}}\right)^{\mathrm{T}}$ and $\mathbf{u}_{\mathrm{R}}^{(k)}=\left(\left(\mathbf{q}_{\mathrm{R}}^{(k)}\right)^{\mathrm{T}}\left(\mathbf{F}_{\mathrm{R}}^{(k)}\right)^{\mathrm{T}}\right)^{\mathrm{T}}$, and that the internal DOFs of substructure $k$ are not submitted to external forces.

According to Mencik and Ichchou (2005), the continuity conditions between the substructures combined with the periodicity condition and the dynamical equilibrium of each substructure can finally lead to the following boundary value problem:

$$
\mathbf{S} \boldsymbol{\Phi}_{i}=\mu_{i} \boldsymbol{\Phi}_{i} \quad, \quad\left|\mathbf{S}-\mu_{i} \mathbf{I}_{\mathbf{2} n}\right|=\mathbf{0} .
$$

where

$$
\mathbf{S}=\left[\begin{array}{c|c}
-\left(\mathbf{D}_{\mathrm{LR}}^{*}\right)^{-1} \mathbf{D}_{\mathrm{LL}}^{*} & -\left(\mathbf{D}_{\mathrm{LR}}^{*}\right)^{-1} \\
\hline \mathbf{D}_{\mathrm{RL}}^{*}-\mathbf{D}_{\mathrm{RR}}^{*}\left(\mathbf{D}_{\mathrm{LR}}^{*}\right)^{-1} \mathbf{D}_{\mathrm{LL}}^{*} & -\mathbf{D}_{\mathrm{RR}}^{*}\left(\mathbf{D}_{\mathrm{LR}}^{*}\right)^{-1}
\end{array}\right]
$$


$\mathbf{D}^{*}$ stands for the dynamical stiffness matrix of substructure $k$, condensed on the DOFs of the left and right boundaries of the substructure. The matrix $\mathbf{I}_{2 n}$ represents the identity matrix of size $2 n$.

As mentioned in reference (Collet et al., 2009), the eigenvalues $\mu_{i}$ and wavenumbers $k_{i}$ are linked through the relation $\mu_{i}=e^{-i k_{i} d_{x}}$, where $d_{x}$ denotes the length of the unit cell in $X$-axis. The sign of the real part of the wavenumber $k_{i}, R e\left(k_{i}\right)$, represents the direction of the phase velocity of the corresponding waves: if $\operatorname{Re}\left(k_{i}\right)>0$, the phase propagates in the positive $x$ direction; if $\operatorname{Re}\left(k_{i}\right)<0$, the phase propagates in the negative direction, and if it is zero, $k_{i}$ corresponds to the wavenumber of a pure evanescent wave that only occurs when an undamped system is considered (Collet et al., 2011). The matrix $\mathbf{\Phi}$ of the eigenvectors can be described in this way:

$$
\Phi=\left[\begin{array}{ll}
\Phi_{\mathrm{q}}^{\text {inc }} & \boldsymbol{\Phi}_{\mathbf{q}}^{\mathrm{ref}} \\
\boldsymbol{\Phi}_{\mathbf{F}}^{\text {inc }} & \boldsymbol{\Phi}_{\mathbf{F}}^{\text {ref }}
\end{array}\right],
$$

where subscripts $\mathbf{q}$ and $\mathbf{F}$ refer to the components which are related to the displacements and the forces, respectively; $\left(\left(\boldsymbol{\Phi}_{\mathbf{q}}^{\text {inc }}\right)^{\mathrm{T}}\left(\boldsymbol{\Phi}_{\mathbf{F}}^{\text {inc }}\right)^{\mathrm{T}}\right)^{\mathrm{T}}$ and $\left(\left(\boldsymbol{\Phi}_{\mathbf{q}}^{\text {ref }}\right)^{\mathrm{T}}\left(\boldsymbol{\Phi}_{\mathbf{F}}^{\text {ref }}\right)^{\mathrm{T}}\right)^{\mathrm{T}}$ stand for the modes which are incident to and reflected by a specific boundary (left or right) of the heterogeneous waveguide, respectively. Finally, assuming modal decomposition, state vectors $\mathbf{u}_{\mathrm{L}}^{(k)}$ and $\mathbf{u}_{\mathrm{R}}^{(k)}$ of any substructures $k$ can be expressed from eigenvectors $\left\{\boldsymbol{\Phi}_{i}\right\}_{i=1, \ldots, 2 n}$ (Zhong and 
Williams, 1995):

$$
\mathbf{u}_{\mathrm{L}}^{(k)}=\boldsymbol{\Phi} \mathbf{Q}^{(k)} \quad, \quad \mathbf{u}_{\mathrm{R}}^{(k)}=\boldsymbol{\Phi} \mathbf{Q}^{(k+1)} \quad \forall k \in\{1, \ldots, N\}
$$

Here, vector $\mathbf{Q}$ stands for the amplitudes of the wave modes. The dynamical behavior of a periodic waveguide can be simply expressed from a basis of modes representing waves traveling in the positive and negative directions of the system. An analysis of the dynamical response consists of evaluating a set of amplitudes $\left\{\left(\mathbf{Q}^{\operatorname{inc}(k)}, \mathbf{Q}^{\text {ref(k) }}\right)\right\}_{k}$ associated with the incident and reflected modes. Nevertheless, to evaluate energy diffusion, the formulation of the coupling conditions of the system is needed, and particularly at a coupling junction where several waveguides can be considered. It should be mentioned that the whole system has free boundary conditions, and the coupling conditions are in fact boundary conditions for subsystems(waveguides and coupling element).

\section{Figure 2}

Let us consider two periodic waveguides which are coupled through a coupling element and let us consider two corresponding substructures (1 and 2) which are located at the ends of the waveguides (see Figure 2). These substructures are coupled with the coupling element at surfaces $\Gamma_{1}$ and $\Gamma_{2}$ and are coupled with the other substructures, into waveguides, at surfaces $S_{1}$ and $S_{2}$. It is assumed that the coupling element is only subject to the 
coupling actions (i.e. there is no force inside the element).

According to Mencik and Ichchou (2005), with the dynamical equilibrium of the wave guides and the coupling element, and their continuity conditions of nodal displacement and force expressed in the modal basis, it can be demonstrated that the dynamical behavior of a given coupled periodic waveguide $i(i=1,2)$ can be simply expressed in terms of wave modes $\left(\left(\boldsymbol{\Phi}_{\mathbf{q}}^{\text {inc }(i)}\right)^{\mathrm{T}}\left(\boldsymbol{\Phi}_{\mathbf{F}}^{\text {inc }(i)}\right)^{\mathrm{T}}\right)^{\mathrm{T}}$ incident to the coupling element and wave modes $\left(\left(\boldsymbol{\Phi}_{\mathbf{q}}^{\text {ref }(i)}\right)^{\mathrm{T}}\left(\boldsymbol{\Phi}_{\mathbf{F}}^{\text {ref }(i)}\right)^{\mathrm{T}}\right)^{\mathrm{T}}$ reflected by the coupling element. It can also be proved that the modal amplitudes $\left(\mathbf{Q}^{\operatorname{ref}(1)}, \mathbf{Q}^{\operatorname{ref}(2)}\right)$ of the modes reflected by the coupling element can be related to amplitudes $\left(\mathbf{Q}^{\text {inc(1) }}, \mathbf{Q}^{\text {inc(2) }}\right)$ of the modes incident to the coupling element via a diffusion matrix, namely $\mathbb{C}$, which relates the reflection and transmission coefficients of the wave modes through the dynamical behavior of the coupling element:

$$
\left(\begin{array}{c}
\mathrm{Q}^{\mathrm{ref}(1)} \\
\mathrm{Q}^{\mathrm{ref}(2)}
\end{array}\right)=\mathbb{C}\left(\begin{array}{c}
\mathrm{Q}^{\mathrm{inc}(1)} \\
\mathrm{Q}^{\mathrm{inc}(2)}
\end{array}\right)
$$

The diffusion matrix $\mathbb{C}$ directly depends on the normalization of eigenvectors $\left\{\boldsymbol{\Phi}_{j}^{(1)}\right\}_{j}$ and $\left\{\boldsymbol{\Phi}_{k}^{(2)}\right\}_{k}$. It seems advantageous to normalize the eigenvectors of the two waveguides in a similar manner. When there is only one unit incident wave mode from waveguide 1 , and assuming that there is no incident wave from waveguide 2 , we have $\mathbf{Q}^{\text {inc(1) }}=\left\{\begin{array}{llllllll}0 & 0 & \ldots & 0 & 1 & 0 & \ldots & 0\end{array}\right\}^{T}$ and $\mathbf{Q}^{\text {inc(2) }}=$ $\{\mathbf{0}\}$, and the reflection and transmission coefficients $\mathbf{C}^{\text {ref }}$ and $\mathbf{C}^{\text {trans }}$ of a 
specific incident mode $i$ can be calculated in the following manner:

$$
\begin{aligned}
\mathbf{C}_{i}^{\text {ref }} & =\mathbf{Q}_{i}^{\text {ref }(1)} \\
\mathbf{C}_{i}^{\text {trans }} & =\mathbf{Q}_{i}^{\text {ref }(2)}
\end{aligned}
$$

\subsection{Modified wave finite element formulation}

The MWFE formulations are firstly given in the work of Mencik and Ichchou (2008) with etailed deductions and discussions. Essential formulations and deductions are extracted and presented below in this subsection.

\subsubsection{Associated substructuring scheme}

We consider a typical multi-layered unit cell, say sub system $k$, belonging to a composite structure(see Figure 3).

\section{Figure 3}

In the present work, the unit cell representative of the composite waveguide is assumed to represent a set of $M$ connected straight homogeneous layers, whose left and right boundaries are denoted, respectively, as $\left\{S_{\mathrm{L}}^{i}\right\}$ and $\left\{S_{\mathrm{R}}^{i}\right\}$ : for each layer $i(i=1, \ldots, M)$, the surfaces $\left\{S_{\mathrm{L}}\right\}$ and are assumed to contain the same number of DOFs, say $n^{i}$. Let us denote as $\Gamma^{i}$ the coupling interface between each layer $i$ and its surroundings, that is, the set of coupled layers

$\{j\}_{j \neq i} \cdot \mathbf{q}^{i}$ and $\mathbf{F}^{i}$ are the displacements and the forces applied to the uncoupled layer on $\left\{S_{\mathrm{L}}^{i}\right\} \cup\left\{S_{\mathrm{R}}^{i}\right\}$.

Following what was previously presented for the WFE approach, the dynamic equilibrium equation of the unit cell is reformulated into a state vector 
representation:

$$
\mathcal{D}\left(\begin{array}{c}
\mathbf{q}_{\mathrm{L}}^{1} \\
\vdots \\
\mathbf{q}_{\mathrm{L}}^{M} \\
\hline \mathbf{q}_{\mathrm{I}}^{1} \\
\vdots \\
\mathbf{q}_{\mathrm{I}}^{M} \\
\hline \mathbf{q}_{\mathrm{R}}^{1} \\
\vdots \\
\mathbf{q}_{\mathrm{R}}^{M}
\end{array}\right)=\left(\begin{array}{c}
\mathcal{F}_{\mathrm{L}}^{1} \\
\vdots \\
\mathcal{F}_{\mathrm{L}}^{M} \\
\mathcal{F}_{\mathrm{I}}^{1} \\
\vdots \\
\mathcal{F}_{\mathrm{I}}^{M} \\
\mathcal{F}_{\mathrm{R}}^{1} \\
\vdots \\
\mathcal{F}_{\mathrm{R}}^{M}
\end{array}\right)=\mathcal{G}\left(\begin{array}{c}
\mathbf{F}_{\mathrm{L}}^{1} \\
\vdots \\
\mathbf{F}_{\mathrm{L}}^{M} \\
\mathbf{F}_{\mathrm{I}}^{1} \\
\vdots \\
\mathbf{F}_{\mathrm{I}}^{M} \\
\mathbf{F}_{\mathrm{R}}^{1} \\
\vdots \\
\mathbf{F}_{\mathrm{R}}^{M}
\end{array}\right),
$$

$\mathcal{D}$ represents the dynamic stiffness matrix, and $\mathcal{G}$ imposes the continuity conditions of the forces between the uncoupled layers Mencik and Ichchou (2008). Assuming that the internal DOFs of the subsystem are not submitted to external forces, that is $\mathcal{F}_{\mathrm{I}}^{i}=\mathbf{0} \forall i$, leads to the following condensed state vector representations for the associated substructuring scheme:

$$
\left(\begin{array}{c}
\mathbf{q}_{\mathrm{R}}^{1} \\
\vdots \\
\mathbf{q}_{\mathrm{R}}^{M} \\
\mathcal{F}_{\mathrm{R}}^{1} \\
\vdots \\
\mathcal{F}_{\mathrm{R}}^{M}
\end{array}\right)=\mathcal{S}\left(\begin{array}{c}
\mathbf{q}_{\mathrm{L}}^{1} \\
\vdots \\
\frac{\mathbf{q}_{\mathrm{L}}^{M}}{} \\
-\mathcal{F}_{\mathrm{L}}^{1} \\
\vdots \\
-\mathcal{F}_{\mathrm{L}}^{M}
\end{array}\right)
$$

Here, $\mathcal{S}$ is a $\left(2 \sum_{i} n^{i} \times 2 \sum_{i} n^{i}\right)$ matrix, expressed by

$$
\mathcal{S}=\left[\begin{array}{cc}
-\left(\mathcal{D}_{\mathrm{LR}}^{*}\right)^{-1} \mathcal{D}_{\mathrm{LL}}^{*} & -\left(\mathcal{D}_{\mathrm{LR}}^{*}\right)^{-1} \\
\mathcal{D}_{\mathrm{RL}}^{*}-\mathcal{D}_{\mathrm{RR}}^{*}\left(\mathcal{D}_{\mathrm{LR}}^{*}\right)^{-1} \mathcal{D}_{\mathrm{LL}}^{*} & -\mathcal{D}_{\mathrm{RR}}^{*}\left(\mathcal{D}_{\mathrm{LR}}^{*}\right)^{-1}
\end{array}\right]
$$


$\mathcal{D}^{*}$ represents the dynamic stiffness matrix condensed on the DOFs of the left and right boundaries. More over, observing that, for each layer $i$, the contribution of the surroundings $\Theta^{i}$ to the forces applied on a specific boundary, either $S_{\mathrm{L}}^{i}$ or $S_{\mathrm{R}}^{i}$, is null except on the boundary $S_{\mathrm{L}}^{\Theta^{i}}$ or $S_{\mathrm{R}}^{\Theta^{i}}$, respectively, leads to the following relationships:

$$
\left(\begin{array}{c}
\mathcal{F}_{\mathrm{R}}^{1} \\
\vdots \\
\mathcal{F}_{\mathrm{R}}^{M}
\end{array}\right)=\mathcal{G}^{*}\left(\begin{array}{c}
\mathbf{F}_{\mathrm{R}}^{1} \\
\vdots \\
\mathbf{F}_{\mathrm{R}}^{M}
\end{array}\right) \quad\left(\begin{array}{c}
-\mathcal{F}_{\mathrm{L}}^{1} \\
\vdots \\
-\mathcal{F}_{\mathrm{L}}^{M}
\end{array}\right)=\mathcal{G}^{*}\left(\begin{array}{c}
-\mathbf{F}_{\mathrm{L}}^{1} \\
\vdots \\
-\mathbf{F}_{\mathrm{L}}^{M}
\end{array}\right)
$$

where $\mathcal{G}^{*}=\mathcal{G}_{\mathrm{LL}}=\mathcal{G}_{\mathrm{RR}}$. The substructuring scheme provided by equations (8) and (10) is interesting compared to the classic model as it allows the local kinematic variables (displacements and forces) of each layer cross-section to be considered independently from the surroundings. Specifically, the method appears interesting for constructing the global wave modes of the multilayered system from a set of local wave mode bases attached to the layers with free interfaces $\left\{\Gamma^{i}\right\}_{i}$ and whose dimensions can be individually tuned to "fit" with each cross-section dynamics. This constitutes the framework of the MWFE formulation presented below. 


\subsubsection{Construction of the global wave mode basis}

Considering the coupling conditions between two consecutive subsystems $k-$ 1 and $k$, established for each layer $i$

$$
\begin{aligned}
\mathbf{q}_{\mathrm{L}}^{i(k)}=\mathrm{q}_{\mathrm{R}}^{i(k-1)} \quad \mathcal{F}_{\mathrm{L}}^{i(k)}=\mathcal{F}_{\mathrm{R}}^{i(k-1)} \\
k=2, \ldots, N \quad i=1, \ldots, M
\end{aligned}
$$

and considering, according to Bloch's theorem Brillouin (1946), wave solutions $\left\{\boldsymbol{\Phi}_{j}\right\}_{j}$ in equation (8) leads to the following eigenvalue problem

$$
\mathcal{S}\left(\begin{array}{c}
\left(\boldsymbol{\Phi}_{\mathbf{q}}\right)_{j \mid 1} \\
\vdots \\
\left(\boldsymbol{\Phi}_{\mathbf{q}}\right)_{j \mid M} \\
\\
\left(\boldsymbol{\Phi}_{\mathcal{F}}\right)_{j \mid 1} \\
\vdots \\
\left(\boldsymbol{\Phi}_{\mathcal{F}}\right)_{j \mid M}
\end{array}\right)=\mu_{j}\left(\begin{array}{c}
\left(\boldsymbol{\Phi}_{\mathbf{q}}\right)_{j \mid 1} \\
\vdots \\
\left(\boldsymbol{\Phi}_{\mathbf{q}}\right)_{j \mid M} \\
\\
\left(\boldsymbol{\Phi}_{\mathcal{F}}\right)_{j \mid 1} \\
\vdots \\
\left(\boldsymbol{\Phi}_{\mathcal{F}}\right)_{j \mid M}
\end{array}\right), \quad\left|\mathcal{S}-\mu_{j} \mathbf{I}\right|=0
$$

where $\left(\boldsymbol{\Phi}_{\mathrm{q}}\right)_{j \mid i}$ and $\left(\boldsymbol{\Phi}_{\mathcal{F}}\right)_{j \mid i}$ are $\left(n^{i} \times 1\right)$ vectors which represent the restriction of the $(n \times 1)$ global mode components $\left(\boldsymbol{\Phi}_{\mathrm{q}}\right)_{j}$ and $\left(\boldsymbol{\Phi}_{\mathrm{F}}\right)_{j}$ to $S_{L}^{i}$ or $S_{R}^{i}$.

The construction of the global wave modes $\left\{\boldsymbol{\Phi}_{j}\right\}_{j}$ can be established in the following matrix form:

$$
\left(\begin{array}{c}
\left(\Phi_{\mathrm{q}}\right)_{j \mid 1} \\
\vdots \\
\left(\boldsymbol{\Phi}_{\mathrm{q}}\right)_{j \mid M} \\
\\
\left(\boldsymbol{\Phi}_{\mathcal{F}}\right)_{j \mid 1} \\
\vdots \\
\left(\boldsymbol{\Phi}_{\mathcal{F}}\right)_{j \mid M}
\end{array}\right)=\boldsymbol{B a}_{j}
$$


Hereafter, the global wave modes $\left\{\boldsymbol{\Phi}_{j}\right\}_{j}$ of the multi-layered system are constructed from a set of reduced local bases

$\left\{\left\{\tilde{\boldsymbol{\Phi}}_{j}^{i}\right\}_{j=1, \ldots, 2 m^{i}}\right\}_{i}=\left\{\left\{\boldsymbol{\Phi}_{j}^{1}\right\}_{j},\left\{\boldsymbol{\Phi}_{k}^{2}\right\}_{k}, \ldots,\left\{\boldsymbol{\Phi}_{l}^{M}\right\}_{l}\right\}$ attached to the homogeneous uncoupled layers and having specific dimensions $\left\{2 m^{i}\right\}_{i}$. Two different modelings for the global wave mode construction are described below: the first MWFE modeling enforces the convergence of the wave mode expansion used in the global wave mode construction, while the second MWFE modeling is based on a relatively well-conditioned eigenvalue problem Mencik and Ichchou (2008).

In the first MWFE modeling, the matrix $\mathcal{B}$ is written as:

$$
\mathcal{B}=\left[\begin{array}{cc}
\mathbf{I} & 0 \\
\mathbf{0} & \mathcal{G}^{*}
\end{array}\right]\left[\begin{array}{l}
\tilde{\boldsymbol{\Phi}}_{\mathrm{q}}^{\text {local }} \\
\tilde{\boldsymbol{\Phi}}_{\mathrm{F}}^{\text {local }}
\end{array}\right]
$$

while in the second MWFE modeling, the matrix $\mathcal{B}$ is expressed in the following form:

$$
\mathcal{B}=\left[\begin{array}{l}
\tilde{\boldsymbol{\Phi}}_{\mathrm{q}}^{\text {local }} \\
\tilde{\boldsymbol{\Phi}}_{\mathrm{F}}^{\text {local }}
\end{array}\right] .
$$

Thus, the set of modal participations $\left\{\mathbf{a}_{j}\right\}_{j}$ can be evaluated by solving a square $\left(2 \sum_{i} m^{i} \times 2 \sum_{i} m^{i}\right)$ classic eigenvalue problem

$$
\mathbf{S}^{\mathrm{MWFE}} \mathbf{a}_{j}=\mu_{j} \mathbf{a}_{j}, \quad\left|\mathbf{S}^{\mathrm{MWFE}}-\mu_{j} \mathbf{I}\right|=0
$$


where

$$
\mathbf{S}^{\mathrm{MWFE}}=\left[\mathcal{B}^{\mathrm{T}} \mathbf{J} \mathcal{B}\right]^{-1} \mathcal{B}^{\mathrm{T}} \mathbf{J} \mathcal{S B}
$$

The eigenvalue problem (16) in central to the MWFE formulation. Given a

set of reduced local bases $\left\{\left\{\tilde{\boldsymbol{\Phi}}_{j}^{i}\right\}_{j}\right\}_{i}$ having appropriate dimensions, the formulation consists of finding the eigenvalues $\left\{\mu_{j}\right\}_{j}$, which describe the global wave mode velocities, and the eigenvectors $\left\{\mathbf{a}_{j}\right\}_{j}$, providing, by means of equation (12), the restrictions of the global wave mode shapes, say $\left\{\left\{\boldsymbol{\Phi}_{j \mid i}\right\}_{i}\right\}_{j}$, to the set of surfaces $\left\{S_{\mathrm{L}}^{i}\right\}$ or $\left\{S_{\mathrm{R}}^{i}\right\}$.

\subsection{Piezoelectric finite element formulation for the cou- pling element}

In the Diffusion Matrix Model(DMM) (Ichchou et al., 2009), in order to consider properly a coupling element with shunted piezoelectric patches, an appropriate formulation should be used. A finite element model of the coupled system consisting of a beam and a pair of identic piezoelectric patches with shunted circuit can established, as displayed in Figure 4.

\section{Figure 4}

This model contains two multi-layered waveguides with 3D linear brick finite elements and a coupling element with 3D linear brick piezoelectric finite elements. The piezoelectric element has 8 nodes and 4 degrees of freedom(DOF) 
per node. Each node has 3 structural DOF and 1 electrical DOF (electrical potential). All electrical potential DOF that are placed on electrode surfaces of the patches are reduced such that only one potential master DOF remains on each piezoelectric patch. All electrical potential DOF on the patch surfaces bonded to the beam are grounded. The whole structure has free mechanical boundary conditions. For the sake of simplicity, detailed deductions of strain energy, mass and stiffness matrices for the piezoelectric elements (without shunt circuit), which can be found in the work of Casadei et al. (2010), are not presented in this paper. The discretized electro-elastic system of equations can be written in the form shown in Equation (18a) and Equation (18b).

$$
\begin{array}{r}
\mathbf{M}_{d d} \ddot{\mathbf{d}}+\mathbf{K}_{d d} \mathbf{d}+\mathbf{K}_{d v} \mathbf{v}=\mathbf{f} \\
\mathbf{K}_{d v}^{T} \mathbf{d}+\mathbf{K}_{v v} \mathbf{v}=\mathcal{Q}
\end{array}
$$

where $\mathbf{d}$ and $\mathbf{v}$ represent the structural and electrical DOF respectively, and:

$$
\begin{aligned}
& \mathbf{M}_{d d}=\int_{V_{s}} \mathbf{N}_{d}^{T} \rho \mathbf{N}_{d} d V, \quad \mathbf{K}_{d d}=\int_{V_{s}} \mathbf{B}_{d}^{T} \mathbf{c}^{E} \mathbf{B}_{d} d V, \quad \mathbf{K}_{d v}=\int_{V_{s}} \mathbf{B}_{d}^{T} \mathbf{e}^{T} \mathbf{B}_{v} d V \\
& \mathbf{K}_{v v}=-\int_{V_{s}} \mathbf{B}_{v}^{T} \boldsymbol{\epsilon}^{S} \mathbf{B}_{v} d V, \quad \mathbf{f}=\int_{S_{f}} \mathbf{N}_{d}^{T} f d S, \quad \mathcal{Q}=-\int_{S_{q}} \mathbf{N}_{v}^{T} q d S .
\end{aligned}
$$

in which $\mathbf{N}_{d}$ and $\mathbf{N}_{v}$ are the shape functions, $\mathbf{B}_{d}=\mathcal{D}^{p} \mathbf{N}_{d}$ and $\mathbf{B}_{v}=\nabla \mathbf{N}_{v}$. $\mathcal{D}^{p}$ is the linear differential operator matrix which relates the strains to the structural displacements $\mathbf{U}$. In this case, the matrix $\mathcal{D}^{p}$ is given in Equa- 
tion (20).

$$
\mathcal{D}^{p}=\left(\begin{array}{ccc}
\frac{\partial}{\partial x} & 0 & 0 \\
0 & \frac{\partial}{\partial y} & 0 \\
0 & 0 & \frac{\partial}{\partial z} \\
\frac{\partial}{\partial y} & \frac{\partial}{\partial x} & 0 \\
0 & \frac{\partial}{\partial z} & \frac{\partial}{\partial y} \\
\frac{\partial}{\partial z} & 0 & \frac{\partial}{\partial x}
\end{array}\right)
$$

After finite element assembly, the discretized coupled piezoelectric and structural field equations are finally given in terms of nodal displacements $\mathbf{U}$ and nodal electric potential V. Following the electrode definitions mentioned in the work of Becker et al. (2006), the electrical potential DOF in the piezoelectric patches are partitioned into three different groups:

- For nodes on the outer surfaces of the piezoelectric patches, their associated electrical DOF are called $\mathbf{V}_{p}$, and they have the same electrical potential;

- For nodes on the inner surfaces of the piezoelectric patches bonded to the beam, their associated electrical DOF are called $\mathbf{V}_{g}$, and they are grounded;

- For nodes inside the piezoelectric patches, their associated electrical DOF are called $\mathbf{V}_{i}$. 
The equations of motion are subsequently written in the form shown in Equation (21).

$$
\left[\begin{array}{cccc}
\mathbf{M}_{u u} & 0 & 0 & 0 \\
0 & 0 & 0 & 0 \\
0 & 0 & 0 & 0 \\
0 & 0 & 0 & 0
\end{array}\right]\left[\begin{array}{c}
\ddot{\mathbf{U}} \\
\ddot{\mathbf{V}}_{i} \\
\ddot{\mathbf{V}}_{p} \\
\ddot{\mathbf{V}}_{g}
\end{array}\right]+\left[\begin{array}{cccc}
\mathbf{K}_{u u} & \mathbf{K}_{u i} & \mathbf{K}_{u p} & \mathbf{K}_{u g} \\
\mathbf{K}_{u i}^{T} & \mathbf{K}_{i i} & \mathbf{K}_{i p} & \mathbf{K}_{i g} \\
\mathbf{K}_{u p}^{T} & \mathbf{K}_{i p}^{T} & \mathbf{K}_{p p} & \mathbf{K}_{p g} \\
\mathbf{K}_{u g}^{T} & \mathbf{K}_{i g}^{T} & \mathbf{K}_{p g}^{T} & \mathbf{K}_{g g}
\end{array}\right]\left[\begin{array}{c}
\mathbf{U} \\
\mathbf{V}_{i} \\
\mathbf{V}_{p} \\
\mathbf{V}_{g}
\end{array}\right]=\left[\begin{array}{c}
\mathbf{F} \\
\mathcal{Q}_{i} \\
\mathcal{Q}_{p} \\
\mathcal{Q}_{g}
\end{array}\right]
$$

As $\mathbf{V}_{g}=0$, the fourth equation and fourth column in the mass and stiffness matrices can be eliminated. Internal potential DOF can be determined by exact static condensation from Equation (21) since internal electric charges $\mathcal{Q}_{i}=0$ :

$$
\mathbf{V}_{i}=-\mathbf{K}_{i i}^{-1} \mathbf{K}_{u i}^{T} \mathbf{U}-\mathbf{K}_{i i}^{-1} \mathbf{K}_{i p} \mathbf{V}_{p}
$$

Since all the nodes on the potential electrode surfaces have identical potentials, an explicit transformation matrix $\mathbf{T}_{\mathbf{m}}$ can be used to define the master potential DOF $\mathbf{V}_{m}$, as shown in Equation (23).

$$
\mathbf{V}_{p}=\mathbf{T}_{\mathbf{m}} \mathbf{V}_{m}
$$

The use of Equation (23) yields the fully coupled dynamics:

$$
\left[\begin{array}{cc}
\mathbf{M}_{u u} & 0 \\
0 & 0
\end{array}\right]\left[\begin{array}{c}
\ddot{\mathbf{U}} \\
\ddot{\mathbf{V}}_{m}
\end{array}\right]+\left[\begin{array}{cc}
\mathbf{H}_{u u} & \mathbf{H}_{u p} \\
\mathbf{H}_{u p}^{T} & \mathbf{H}_{p p}
\end{array}\right]\left[\begin{array}{c}
\mathbf{U} \\
\mathbf{V}_{m}
\end{array}\right]=\left[\begin{array}{c}
\mathbf{F} \\
\mathcal{Q}_{m}
\end{array}\right]
$$


with

$$
\begin{aligned}
\mathbf{H}_{u u} & =\mathbf{K}_{u u}-\mathbf{K}_{u i} \mathbf{K}_{i i}^{-1} \mathbf{K}_{u i}^{T} \\
\mathbf{H}_{u p} & =\left(\mathbf{K}_{u p}-\mathbf{K}_{u i} \mathbf{K}_{i i}^{-1} \mathbf{K}_{i p}\right) \mathbf{T}_{m} \\
\mathbf{H}_{p p} & =\mathbf{T}_{m}^{T}\left(\mathbf{K}_{p p}-\mathbf{K}_{i p}^{T} \mathbf{K}_{i i}^{-1} \mathbf{K}_{i p}\right) \mathbf{T}_{m} \\
\mathcal{Q}_{m} & =\mathbf{T}_{m}^{T} \mathcal{Q}_{p}
\end{aligned}
$$

After the definition of the master DOF, the R-L shunt circuit can be considered. The electrical impedance of the circuit under harmonic excitation can be written as:

$$
Z_{s h}=R+j \omega L
$$

If there is only one Master electric DOF, $\mathcal{Q}_{m}$ and $V_{m}$ become scalar, thus the current $I_{s h}$ in the shunt circuit can be expressed as Equation (27)

$$
I_{s h}=j \omega \mathcal{Q}_{m}=\frac{V_{m}}{Z_{s h}}
$$

By substituting Equation (27) into Equation (24), the electrical DOF can be condensed and the equation that governs the structural dynamics under harmonic excitation is shown in Equation (28).

$$
\left[\mathbf{H}_{u u}-\omega^{2} \mathbf{M}_{u u}+\mathbf{H}_{u p}\left(\frac{1}{j \omega Z_{s h}}-\mathbf{H}_{p p}\right)^{-1} \mathbf{H}_{u p}^{T}\right] \mathbf{U}=\mathbb{D}^{c i} \mathbf{U}=\mathbf{F}
$$

Equation (28) gives a full finite element description of the shunted piezoelectric patches, as a part of the coupling element for the DMM calculation. 
Matrix $\mathbb{D}^{c i}$ represents the dynamical stiffness matrix of the shunted piezoelectric patch.

\section{NUMERICAL SIMULATIONS OF MULTI- LAYERED COMPOSITE BEAM WITH SHUNTED PIEZOELECTRIC PATCHES}

In this section, firstly the WFE method is applied for the analysis of wave propagation in the multi-layered beam. Then the MWFE formulation is applied to the same structure and results like dispersion curves issued from these two different approaches are compared. The DMM with shunted piezoelectric elements is subsequently employed to calculate the reflection and transmission coefficients of the $Z$-axis bending wave in the multi-layered beam. The influence of the shunted piezoelectric patches on the propagation of this wave mode is carefully investigated with DMM.

\subsection{Dispersion analysis with WFE and MWFE}

The structure to be studied here is a 3-layered beam with 2 identical $R-L$ shunted piezoelectric patches. The finite element model of the waveguide is shown in Figure 5, with the definition of geometric parameters. Numerical values of the parameters are listed in Table 1.

\section{Figure 5}




\section{Table 1}

The material of the outer layers of the multi-layered beam is steel and considered as isotropic, with Young's modulus $E_{b}=210 \mathrm{GPa}$ and Poisson's ratio $\nu_{b}=0.33$, and density $\rho_{b}=7850 \mathrm{~kg} / \mathrm{m}^{3}$. The core of the multi-layered beam is much softer and lighter than the skin layers, with Young's modulus $E_{b}=2 M P a$ and Poisson's ratio $\nu_{b}=0.3$, and density $\rho_{b}=1000 \mathrm{~kg} / \mathrm{m}^{3}$. A loss factor $\eta=0.001$ is added to the finite element model as the system is considered to be dissipative.

Both the WFE and MWFE approaches are applied to the same structure. The analysis is carried out on the frequency band from 0 to $3 \mathrm{kHz}$ so that several cross-section modes can appear. First of all, the dispersion curves of the uncoupled layers are calculated via the WFE approach. The results are shown in Figure 6.

\section{Figure 6}

Later the second MWFE modeling is applied and full wave mode bases of all the 3 uncoupled layers are used. The dimension of the MWFE problem is $\sum_{i} m^{i}=105$, which is considerably larger than the dimension of the WFE $\operatorname{problem}(n=75)$, due to the substructuring technique. By looking at the dispersion curves provided by the second MWFE modeling in Figure 7, it is clear that the two methods give nearly identical results. This validates the underlying substructuring technique of the MWFE formulation.

\section{Figure 7}


Unlike the WFE formulation, the MWFE formulation is able to capture the essential global wave modes of the multi-layered system. For each layer, the chosen local wave modes are expected to significantly contribute to the dynamics of the system. Specifically, for the outer layers in steel, these local wave modes represent rigid cross-section wave motions, while for the soft middle layer, these local wave modes represent not only rigid crosssection wave motions but also a set of contributing cross-section modes. The first MWFE modeling is used to construct the global wave modes, from the set of reduced wave mode bases attached to the uncoupled layers, with $m^{1}=m^{3}=4$ and $m^{2}=30$. Now the dimension of the MWFE problem is $\sum_{i} m^{i}=38$, which appears much smaller than the dimension of the WFE $\operatorname{problem}(n=75)$. The dispersion curves associated with the global wave modes are displayed in Figure 8.

\section{Figure 8}

It can be noticed from Figure 8 that for the 4 rigid cross-section modes (Modes 1 to 4) and the shearing mode (Mode 5), MWFE and WFE give similar results; however, for the other 3 cross-section modes (Modes 6 to 8), with the MWFE method, their cutting-on frequencies are generally smaller than those obtained with the WFE method.

It should also be noticed that for Mode 2 and Mode 7, their dependency at about $2.2 \mathrm{kHz}$ with the WFE method can be removed through the local wave mode basis truncation of the first MWFE formulation. When the sizes of the mode bases overestimate the dynamics of each layer, the mode 
dependency will occur. In this case, the wave mode classification criterion is not capable of distinguishing theses two wave modes around this frequency, as their deformed shapes are similar to each other, as shown in Figures 9 and 10 .

\section{Figure 9}

\section{Figure 10}

It should be mentioned that the deformed shapes are obtained using the second MWFE modeling. The continuity of displacement components at the coupling interfaces is well respected.

The physical wave behavior of the three-layered system is correctly captured with the reduced mode basis, and the gain of computational time is rather evident, which represents another advantage of the MWFE modeling.

\subsection{Energy diffusion analysis with DMM for the $Z$-axis bending mode}

In this section, the energy diffusion problem is dealt with via the DMM proposed in Subsection 2.1.

The three-layered beam is equipped with two identical $R-L$ shunted piezoelectric patches. These piezoelectric patches are fabricated by Saint-Gobain Quartz (type SG P189) and the corresponding material characteristics are

listed in Appendix A. This type of piezoelectric patch works mainly in the 
3-1 mode. The finite element model of the coupling element is displayed in Figure 11. Numerical values of the parameters are listed in Table 1.

\section{Figure 11}

As in this paper, the mostly concerned mode is the $Z$-axis bending wave

mode (Mode 4), and with the MWFE modeling, this mode can already be successfully captured, the dimension of the MWFE problem stays the same for the moment $\left(m^{1}=m^{3}=4\right.$ and $\left.m^{2}=30\right)$. The deformed modal shapes of the $Z$-axis bending mode issued from the WFE and MWFE methods are displayed in Figure 12(a) and Figure 12(b) respectively.

\section{Figure 12}

The reflection and transmission coefficients of the $Z$-axis bending wave mode can be calculated with the DMM of the two different formulations(WFE and MWFE). In the shunt circuit, a resistance $R=10 \Omega$ and an inductance of $L=3 \mathrm{H}$ are used, to obtain a tuning frequency at about $2.2 \mathrm{kHz}$. The tuning frequency can be calculated according to Equation (29):

$$
f_{\text {tune }}=\frac{1}{2 \pi \sqrt{L C_{p 3}^{S}}}
$$

where $C_{p 3}^{S}$ is the capacitance of the piezoelectric patch measured at constant strain. The subscript 1 represents the $X$-axis direction while the subscript 3 
denotes the $Z$-axis direction. It can be calculated in the following manner:

$$
C_{p 3}^{S}=\frac{\epsilon^{S} \times A_{3}}{L_{3}}
$$

where $A_{3}=b_{b} \times L_{b}$ is the area of the surface of the piezoelectric patch perpendicular to $Z$-axis, $L_{3}=h_{p}$ is the thickness of the piezoelectric patch in $Z$-axis direction.

For the two different formulations WFE and MWFE, the corresponding DMM are obtained in slightly different ways: with the WFE formulation, the coupling element in the DMM is treated in a traditional manner, with all the structural and electric DOFs condensed to the DOFs at left and right boundaries; however, with the MWFE formulation, the coupling element is treated layer by layer: for the layer with bonded shunted piezoelectric patch, the structural and electric DOFs in the piezoelectric patch are condensed, and only the structural DOFs of the layer remain; for the layer not connected to the piezoelectric patches, it is modeled in a general manner. Then all the layers are assembled with the MWFE formulation, and the DOFs in the set of layers are condensed to the DOFs at the left and right boundaries. It should also be mentioned that the diffusion matrix $\mathbb{C}$ depends not only on the dynamics of the coupling element, but also on the wave modes extracted after the calculation of the eigenvalue problem in equation (16) associated to the waveguides. In fact, the dimension of $\mathbb{C}$ depends directly on the number of wave modes retained $n_{c}$ after the calculation of the eigenvalue problem (16) 
$\left(n_{c}<2 \sum_{i} m^{i}\right)$, which can be chosen according to the number of propagating wave modes in the waveguides in the frequency band of interest for example. In the WFE and MWFE approaches, the dimension of the diffusion matrix $\mathbb{C}$ is chosen to be the same $\left(n_{c} \times n_{c}\right)$.

Firstly, the first MWFE formulation is applied, with $m^{1}=m^{3}=4$ and $m^{2}=30$, and results are compared to those issued from the WFE method, as shown in Figure 13.

\section{Figure 13}

It can be noted that generally, the reflection coefficients obtained via the WFE approach are bigger than those issued from the MWFE approach. This fact might be due to the continuity conditions imposed by the two approaches: WFE method used classical finite element model of the coupling element, the layers are bonded together, while the MWFE method used Boolean operators to consider the continuity conditions between the layers, then the coupling element in the WFE approach seems to be more rigid than that in the MWFE approach.

Subsequently, the first MWFE formulation (with $m^{1}=m^{3}=4$ and $m^{2}=30$ ) is used and reflection and transmission coefficients are compared to those issued from the second MWFE formulation (with $m^{1}=m^{3}=30$ and $m^{2}=45$, full mode bases of the 3 layers), as displayed in Figure 14.

\section{Figure 14}


It can be seen from Figure 14 that with the DMM of the first MWFE formulation, greater reflection coefficients are obtained than that of the second MWFE formulation. This might be explained as follows: as in the first MWFE modeling, there are much less wave modes than in the second MWFE modeling, and the dependency of global wave modes is removed thanks to the truncation of local wave mode bases of each uncoupled layer, the energy of the incident $Z$-axis bending wave will not be distributed to high order parasite wave modes. Both in Figure 13 and 14 there are some fluctuations in the transmission coefficients obtained via the first MWFE modeling. This might result from numerical error during the computation. Globally, the first and second MWFE modelings give similar results, especially for the frequency band around the tuning frequency $f=2.2 \mathrm{kHz}$.

\subsection{Parametric studies}

In Subsection 3.1, it has been observed that with the second MWFE formulation, when full modal bases are applied for the layers, it gives nearly the same dispersion curves as the WFE formulation. However, when the first MWFE formulation is applied, as truncated modal bases of the layers are applied, this method gives different results from the WFE formulation. For the cross-section modes (Modes 6 to 8 in Figure 8), the cut-on frequencies predicted with MWFE formulation and WFE formulation are quite distinct. Additionally, as mentioned in Subsection 3.2, with the MWFE formulations, 
fluctuations in the transmission coefficients can be noted. In order to understand the influence of the parameters applied in the MWFE modeling on the prediction of dispersion curves and diffusion coefficients, and at the same time try to improve the quality of the numerical results, it's interesting to perform several parametric studies.

\subsubsection{Parametric studies on dispersion curves}

In Subsection 3.1, with the first MWFE modeling, the sizes of the modal basis of each layer are $m^{1}=m^{3}=4$ and $m^{2}=30$, and the dimension of the MWFE problem is $\sum_{i} m^{i}=38$. If larger modal basis is used for the outer layers 1 and 3, for example, $m^{1}=m^{3}=6$ and $m^{2}=30$, the results are closer to WFE results, as displayed in Figure 15.

\section{Figure 15}

The cut-on frequency of Mode 8 calculated with the new wave mode bases using the first MWFE method is almost the same as that calculated with WFE method. However, for the cut-on frequency of Mode 7, there is always a difference between the two methods. When the sizes of the modal bases are applied in the second MWFE modeling, similar results can be found, except for Mode 2 and Mode 7, as the mode dependency occurs between these two modes, as displayed in Figure 16.

Figure 16 
If even larger wave mode bases are used in the second MWFE formulation, for example, $m^{1}=m^{3}=6$ and $m^{2}=45$, MWFE results converges to WFE results. In this case, full mode wave basis is applied for Layer 2. The dispersion curves are shown in Figure 17.

\section{Figure 17}

It can be concluded from the previous results that the sizes of the mode basis of Layers 1 and 3 have a direct influence on the Mode 8, while the size of the mode basis of Layer 2 has an effect on the Mode 7. The deformed shape of Mode 8 is given in Figure 18.

\section{Figure 18}

The other cross-section modes (Mode 5 and Mode 6) can be correctly captured in all cases with proper mode basis sizes. With the MWFE modeling, dependent wave modes should be avoided in the mode bases of the 3 layers in order to guaranteer the convergence of this method, thus the size of the mode basis of each layer should not be too large so as not to overestimate the dynamics of each layer.

\subsubsection{Parametric studies on diffusion coefficients}

Subsequently, a set of mode bases of different sizes are given in Table 2 . The effect of the mode basis size on the calculation of the reflection and transmission coefficients of the bending mode in $Z$-axis (Mode 4 ) is then investigated in detail. 


\section{Table 2}

Firstly, the influence of the dimension of the inner layer (Layer 2) is analyzed. Mode bases 1, 2 and 3 are used, as the dimension of the mode bases in layers 1 and $3 m^{1}=m^{3}=6$, and that of the layer $2 \mathrm{~m}^{2}$ varies from 30 to 45. It should be mentioned that $m^{1}$ larger than 6 is never used as in the frequency band of interest, the number of propagating modes is 4 . Only the 4 propagating modes and 2 evanescent modes $(X$-axis torsion and $Z$-axis bending) are retained as the imaginary parts of their wavenumbers are not too far away from 0 . The wavenumber of other wave modes are with a large imaginary part and will not be taken into the mode basis. All the wave modes in the mode bases are classified according to the imaginary part of their wavenumber: wavenumber with smaller imaginary part is on the top of the mode basis. The reflection coefficients calculated with the 3 different mode bases using MWFE formulation, as well as those obtained via the WFE approach, are compared in Figure 19.

\section{Figure 19}

It can be seen from Figure 19 that mode basis $1\left(m^{2}=40\right)$ and mode basis $2\left(m^{2}=30\right)$ give nearly the same reflection coefficients as those issued from the WFE method, while mode basis $3\left(m^{2}=45\right)$ results in much lower reflection coefficients. The fluctuations in the transmission coefficients becomes weaker when $m^{2}$ becomes larger. Here, according to this comparison, $m^{2}=30$ is likely to be the best mode basis dimension for layer 2 when 
$m^{1}=m^{3}=6$.

Later the influence of the sizes of the outer layers 1 and 3 is studied. Mode bases 3, 4 and 5 are applied, as the dimension of the mode basis in layer 2 stays the same $m^{2}=45$, and $m^{1}$ varies from 4 to 6 . The reflection coefficients calculated with the 3 different mode bases using MWFE formulation, as well as those obtained via the WFE approach, are compared in Figure 20.

\section{Figure 20}

It can be seen from Figure 20 that with $m^{2}=45$, reflection coefficients obtained via the MWFE approach are generally larger than WFE results, and for the results with mode bases 4 and $5\left(m^{1}=m^{3}=5\right.$ and 4 respectively), the transmission coefficients are not accurate. Here the best mode basis dimension for outer layers 1 and 3 is $m^{1}=m^{3}=6$, when $m^{2}=45$. If the mode basis dimension of the inner layer 2 is changed to $m^{2}=30$, the results are displayed in Figure 21.

\section{Figure 21}

With $m^{2}=30$, the reflection coefficients obtained with mode bases 2,8 and 9 are much closer to WFE results than those acquired with $m^{2}=45$. And with mode basis $2\left(m^{1}=m^{3}=6\right)$, the reflection coefficients are nearly the same as WFE results, and the fluctuation in transmission coefficients is not too strong.

By following the same procedure, if $m^{2}$ is fixed to 40 , and by varying $m^{1}=m^{3}$ 
from 4 to 6 , it is interesting to see from Figure 22 that only with the mode basis $1\left(m^{1}=m^{3}=6\right)$, the transmission coefficients can be acquired correctly.

\section{Figure 22}

The last parametric test is carried out with $m^{1}=m^{3}=5$ and by varying $m^{2}$ from 30 to 45 , using mode bases 6,8 and 10 . The results are given in Figure 23.

\section{Figure 23}

It can be noted that with mode basis $6\left(m^{2}=40\right)$ and mode basis $10\left(m^{2}=\right.$ 36), the transmission coefficients are more accurate. If the evanescent $X$-axis torsion wave ( $5^{\text {th }}$ mode in the mode bases, the $6^{\text {th }}$ being the evanescent $Z$ axis bending wave) is taken into the mode basis of the outer layers 1 and 3 , smaller dimension of the mode basis of layer 2 should be chosen.

From all the parametric studies performed here, it can be concluded that the most important mode basis dimension is $m^{2}$. With a proper $m^{2}$ chosen $\left(m^{2}<\right.$ 40 for example), reflection and transmission coefficients are less sensitive to $m^{1}$ and $m^{3}$. However, if $m^{2}$ is larger and overestimates the dynamics of the inner layer $2, m^{1}$ and $m^{3}$ should be properly chosen to obtain correct results $\left(m^{1}=m^{3}=6\right.$ for example). Generally speaking, larger mode basis dimension for outer layers 1 and 3 tends to give transmission coefficients with less fluctuation. 


\section{CONCLUSIONS}

Multi-layered beams with $R-L$ shunted piezoelectric patches are calculated using the MWFE formulation adjusted to consider piezoelectric elements. The control of energy diffusion parameters of wave modes propagating in such composite beams has been studied with two different kinds of MWFE formulation and the associated DMM approach. A modal reduction technique has been applied in the MWFE formulation so as not to overestimate the dynamics of the multi-layered system. Pertinent local wave mode bases of the uncoupled layers with correct dimensions should be applied in the MWFE formulation.

Through parametric studies on the dimensions of local wave mode bases in the MWFE formulations, several conclusions can be drawn:

- For the analysis of wave dispersion, it can be concluded that the dimensions of the local mode bases of outer Layers 1 and 3 have an impact on Mode 6 and Mode 8, while the dimension of the local mode basis of the inner Layer 2 influences mainly the Mode 7. Mode conversion between Mode 2 and Mode 7 occurs when the dimension of the local mode basis of the inner Layer 2 is too large, or the MWFE formulation itself does not guarantee the continuity of the forces at the interfaces of the uncoupled layers (second MWFE).

- For the analysis of energy diffusion, it can be summarized that the dimension of the local mode basis of Layer 2 is the most important pa- 
rameter. If the dimension of this mode basis doesn't overestimate the dynamics of this layer, reflection coefficients issued from the MWFE formulations are close to those issued from the classical WFE method. Additionally, when the dimension of the local mode basis of Layer 2 is smaller, the reflection and transmission calculated for the $Z$-axis bending wave are less sensitive to dimensions of the local wave mode bases of Layers 1 and 3. These dimensions influence mainly the fluctuation in transmission coefficients.

The formulations developed in this work are general and can be applied for all kinds of slender multi-layered smart composite structures. The numerical techniques presented here enable the evaluation of the performance of shunted piezoelectric patches on the control of wave propagation in multi-layered smart composite beams, and facilitate design modifications and systematic investigations of geometric and electric parameters of multi-layered smart composite beams with shunted piezoelectric patches. The experimental validation of the numerical results will be focused on in future work.

\section{ACKNOWLEDGEMENT}

This work is supported by a collaborative research agreement (ANR NT09 617542) between Georgia Tech, FEMTO-ST Institute and Ecole Centrale de Lyon. We gratefully acknowledge Georgia Tech and the French ANR and CNRS for supporting such international collaborations. 


\section{A Appendix A: material properties of the piezo- electric patch (type SG P189)}

Mass density $\rho: \rho=7650 \mathrm{~kg} / \mathrm{m}^{3}$.

Material stiffness matrix $\mathbf{c}^{E}$ :

$$
\mathbf{c}^{E}=10^{10} \times\left[\begin{array}{cccccc}
15.4 & 8.263 & 7.859 & 0 & 0 & 0 \\
8.263 & 15.4 & 7.859 & 0 & 0 & 0 \\
7.859 & 7.859 & 13.74 & 0 & 0 & 0 \\
0 & 0 & 0 & 4.59 & 0 & 0 \\
0 & 0 & 0 & 0 & 4.59 & 0 \\
0 & 0 & 0 & 0 & 0 & 3.57
\end{array}\right] P a
$$

The piezoelectric stress coupling matrix e:

$$
\mathbf{e}=\left[\begin{array}{cccccc}
0 & 0 & 0 & 0 & 12.88 & 0 \\
0 & 0 & 0 & 12.88 & 0 & 0 \\
-6.187 & -6.187 & 12.80 & 0 & 0 & 0
\end{array}\right] N /(V \cdot m)
$$


The permittivity matrix under constant strain $\epsilon^{S}$ :

$$
\boldsymbol{\epsilon}^{S}=10^{-8} \times\left[\begin{array}{ccc}
1.011 & 0 & 0 \\
0 & 1.011 & 0 \\
0 & 0 & 0.591
\end{array}\right] C /(V \cdot m)
$$

\section{References}

Becker, J., Fein, O., Maess, M., Gaul, L., 2006. Finite element-based analysis of shunted piezoelectric structures for vibration damping. Computers and Structures 84 (31-32), 2340-2350.

Brillouin, L., 1946. Wave propagation in periodic structures. McGraw-Hill, New York.

Casadei, F., Ruzzene, M., Dozio, L., Cunefare, K. A., 2010. Broadband vibration control through periodic arrays of resonant shunts: experimental investigation on plates. Journal of Smart Materials and Structures 19 (1), 015002.

Collet, M., Cunefare, K. A., Ichchou, M. N., 2009. Wave motion optimization in periodically distributed shunted piezocomposite beam structures. Journal of Intelligent Material Systems and Structures 20 (7), 787-808.

Collet, M., Ouisse, M., Ruzzene, M., Ichchou, M. N., 2011. Floquetcbloch decomposition for the computation of dispersion of two-dimensional pe- 
riodic, damped mechanical systems. International Journal of Solids and Structures 48 (20), 2837-2848.

Collet, M., Walter, V., Delobelle, P., 2003. Active damping of a microcantilever piezocomposite beam. Journal of Sound and Vibration 260 (3), 453-476.

Duhamel, D., Mace, B. R., Brennan, M. J., 2006. Finite element analysis of the vibrations of waveguides and periodic structures. Journal of Sound and Vibration 294 (1-2), 205-220.

Hagood, N. W., Flotow, A., 1991. Damping of structural vibrations with piezoelectric materials and passive electrical networks. Journal of Sound and Vibration 146, 243-268.

Huang, T. L., Ichchou, M. N., Bareille, O. A., 2012a. Multi-mode wave propagation in damaged stiffened panels. Structural Control and Health Monitoring 19 (5), 609-629.

Huang, T. L., Ichchou, M. N., Bareille, O. A., Collet, M., Ouisse, M., 2012b. Traveling wave control in thin-walled structures through shunted piezoelectric patches. Mechanical Systems and Signal Processing, doi: 10.1016/j.ymssp.2012.06.014.

Ichchou, M. N., Akrout, S., Mencik, J.-M., 2007. Guided waves group and energy velocities via finite elements. Journal of Sound and Vibration 305, 931-944. 
Ichchou, M. N., Mencik, J.-M., Zhou, W., 2009. Wave finite elements for low and mid-frequency description of coupled structures with damage. Computer methods in applied mechanics and engineering 198 (15-16), 1311 1326.

Mace, B. R., Duhamel, D., Brennan, M. J., Hinke, L., 2005. Finite element prediction of wave motion in structural waveguides. Journal of Acoustical Society of America 117 (5), 2835-2843.

Mace, B. R., Manconi, E., 2008. Modelling wave propagation in twodimensional structures using finite element analysis. Journal of Sound and Vibration 318 (4-5), 884-902.

Mencik, J.-M., Ichchou, M. N., 2005. Multi-mode propagation and diffusion in structures through finite elements. European Journal of Mechanics A/Solids 24 (5), 877-898.

Mencik, J.-M., Ichchou, M. N., 2007. Wave finite elements in guided elastodynamics with internal fluid. International Journal of Solids and Structures 44, 2148-2167.

Mencik, J.-M., Ichchou, M. N., 2008. A substructuring technique for finite element wave propagation in multi-layered systems. Computer Methods in Applied Mechanics and Engineering 197 (6-8), 505-523.

Meyer, Y., Verdot, T., Collet, M., 2007. Active isolation of electronic micro- 
components with piezoelectrically transduced silicon MEMS devices. Journal of Smart Materials and Structures 16 (1), 128-134.

Nguyen, C. H., Pietrzko, S. J., 2006. FE analysis of a PZT-actuated adaptive beam with vibration damping using a parallel R-L shunt circuit. Finite elements in analysis and design 42 (14-15), 1231-1239.

Ohayon, R., Soize, C., 1998. Structural Acoustics and Vibration. Academic Press, San Diego.

Spadoni, A., Ruzzene, M., Cunefare, K. A., 2009. Vibration and wave propagation control of plate with periodic arrays of shunted piezoelectric patches. Journal of Intelligent Material Systems and Structures 20 (8), 979-990.

Thorp, O., Ruzzene, M., Baz, A., 2001. Attenuation and localization of wave propagation in rods with periodic shunted piezoelectric patches. Journal of Smart Materials and Structures 10 (5), 979-989.

Wang, G., Chen, S., Wen, J., 2011. Low-frequency locally resonant band gaps induced by arrays of resonant shunts with antoniou's circuit: experimental investigation on beams. Smart Materials and Structures 20 (1), 015026.

Zhong, W. X., Williams, F., 1995. On the direct solution of wave propagation for repetitive structures. Journal of Sound and Vibration 181 (3), 485-501. 


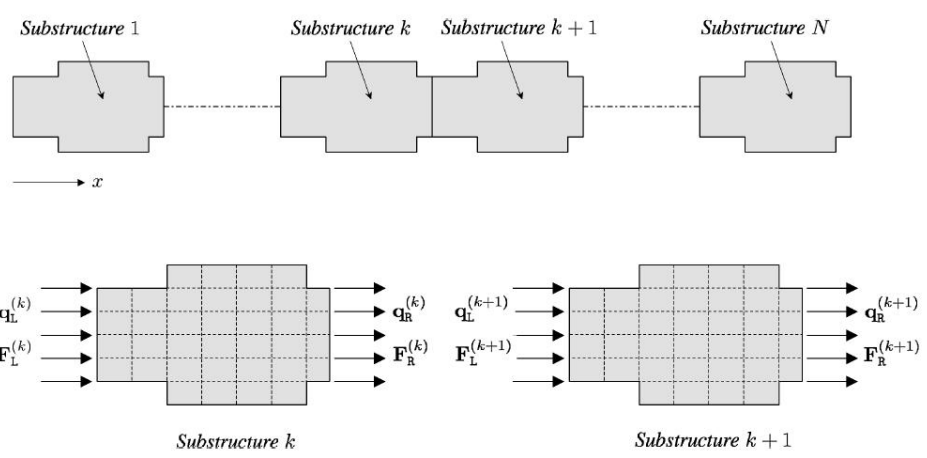

Figure 1: An illustration of a periodic waveguide (Mencik and Ichchou, 2005). 


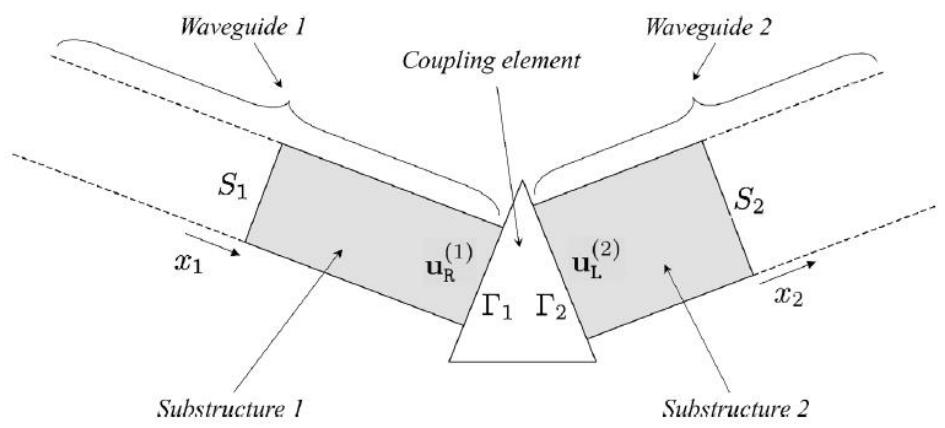

Figure 2: An illustration of the coupling between two different periodic waveguides (Mencik and Ichchou, 2005). 

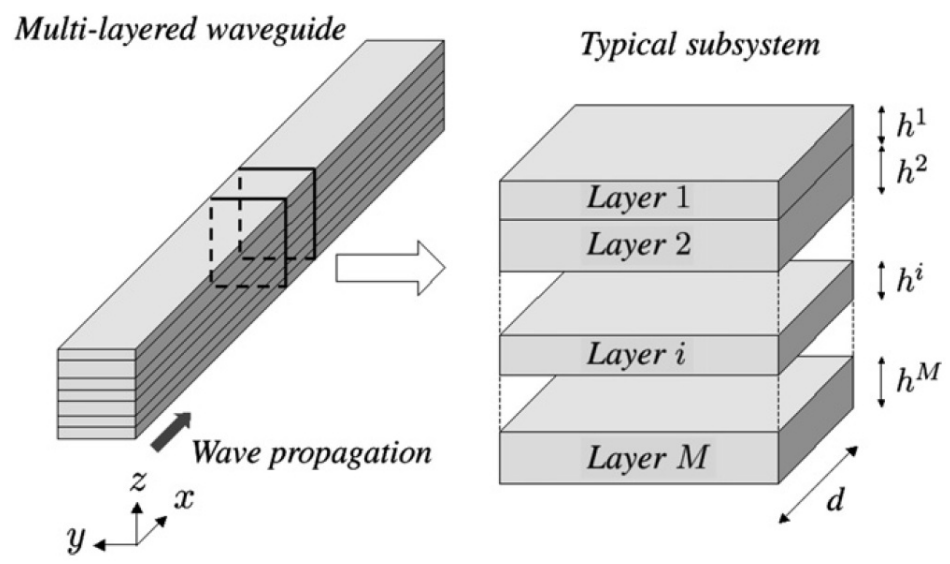

Figure 3: An illustration of a multi-layered elastic system with a rectangular cross-section (Mencik and Ichchou, 2008). 


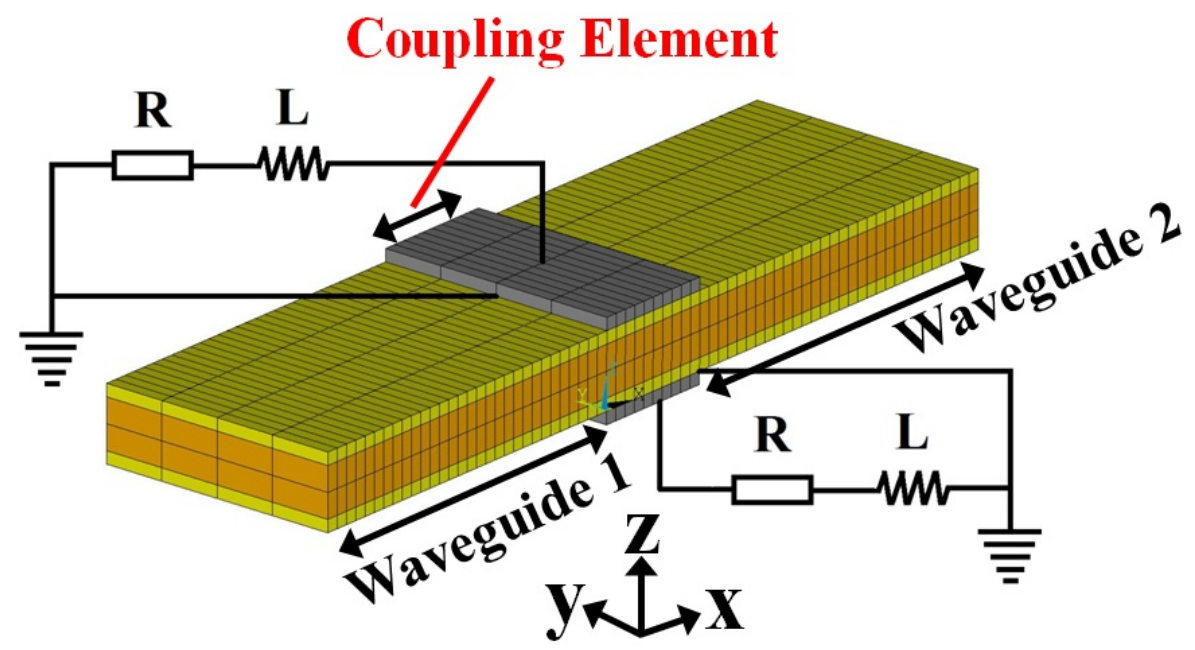

Figure 4: Finite element model of a multi-layered beam with symmetric shunted piezoelectric patches. The coupling element is the part of the beam with the two symmetrically bonded piezoelectric patches. 


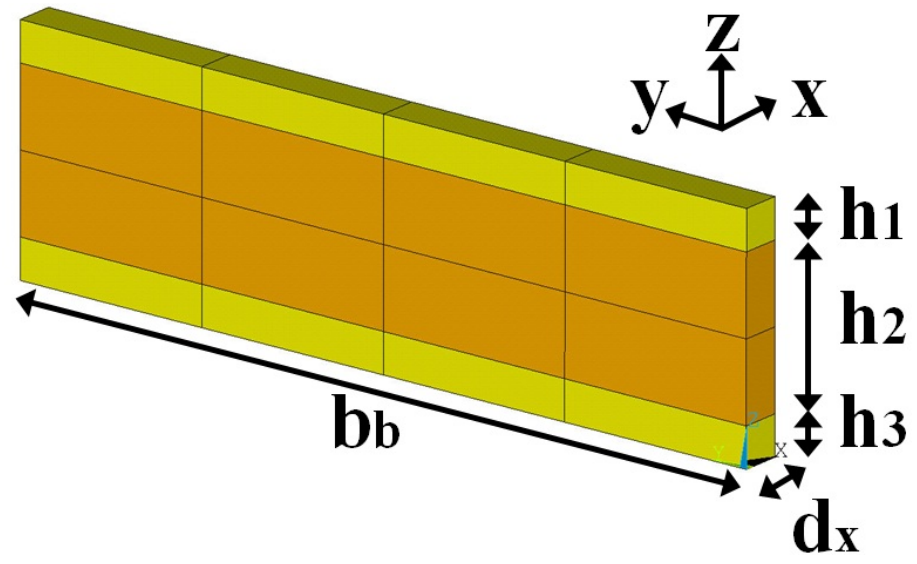

Figure 5: Finite element model of the unit cell representative of the multilayered beam as a waveguide. 


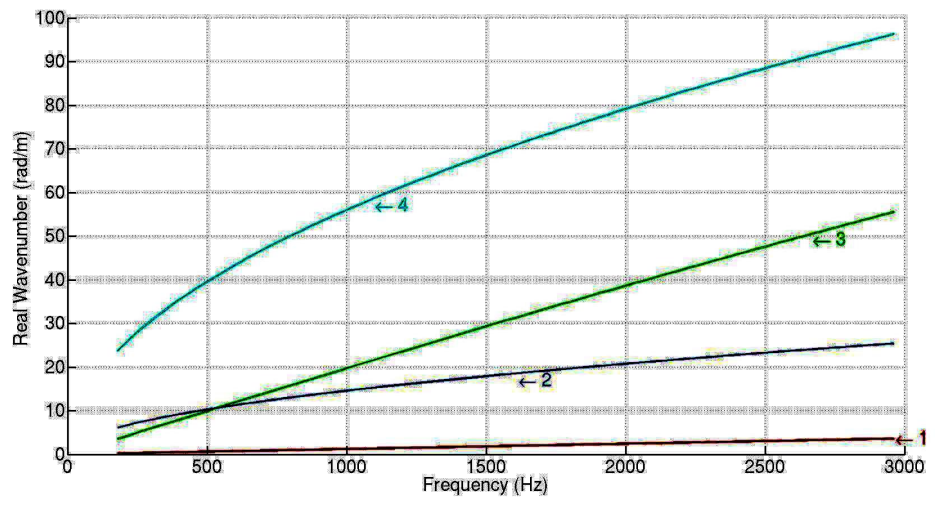

(a) Layers 1 and 3

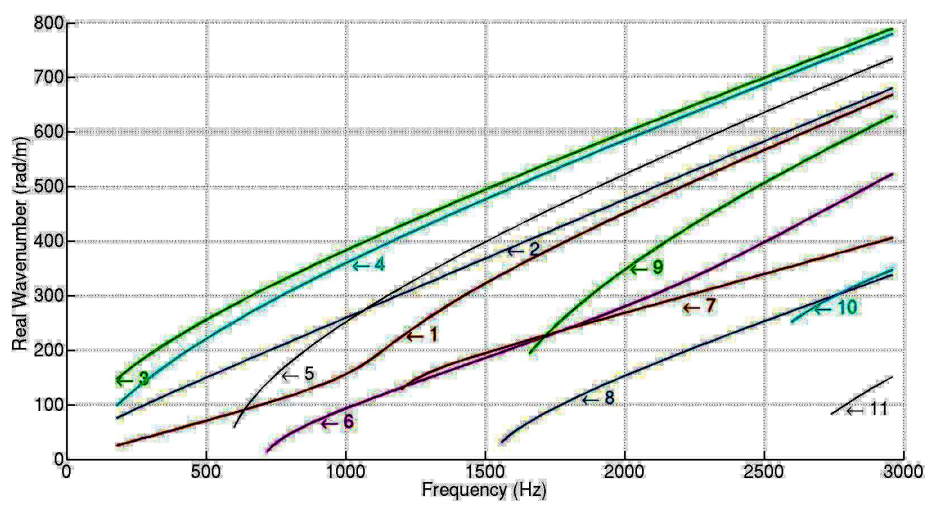

(b) Layer 2

Figure 6: Dispersion curves of wave modes propagating in(a)Layers 1 and 3 (b)Layer 2, in the frequency band from 0 to $3 \mathrm{kHz}$, using the WFE approach. 


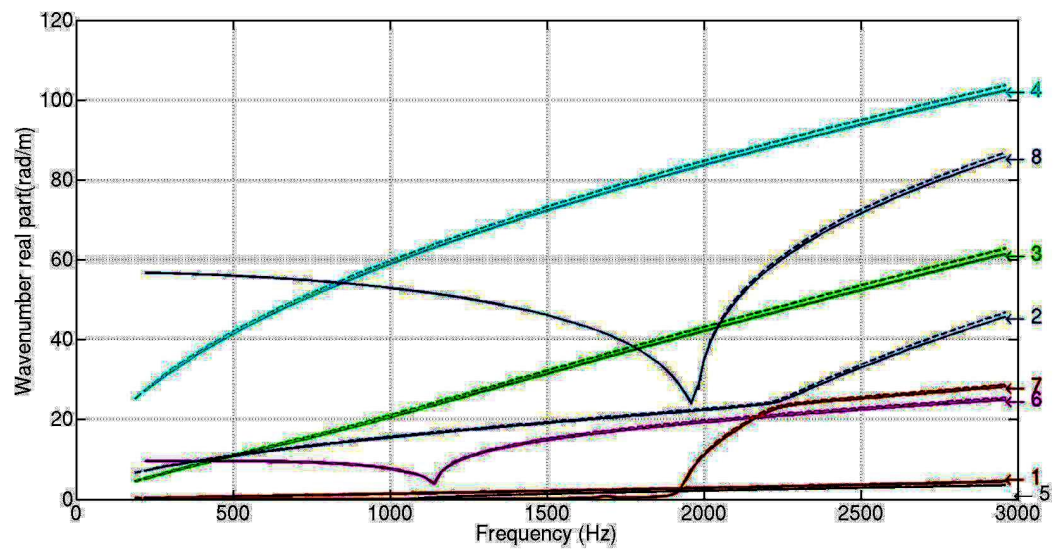

Figure 7: Dispersion curves for the global waveguide obtained using the second MWFE modeling, based on the full wave mode basis of each uncoupled layer. (solid lines)MWFE results (dashed lines)WFE results. 


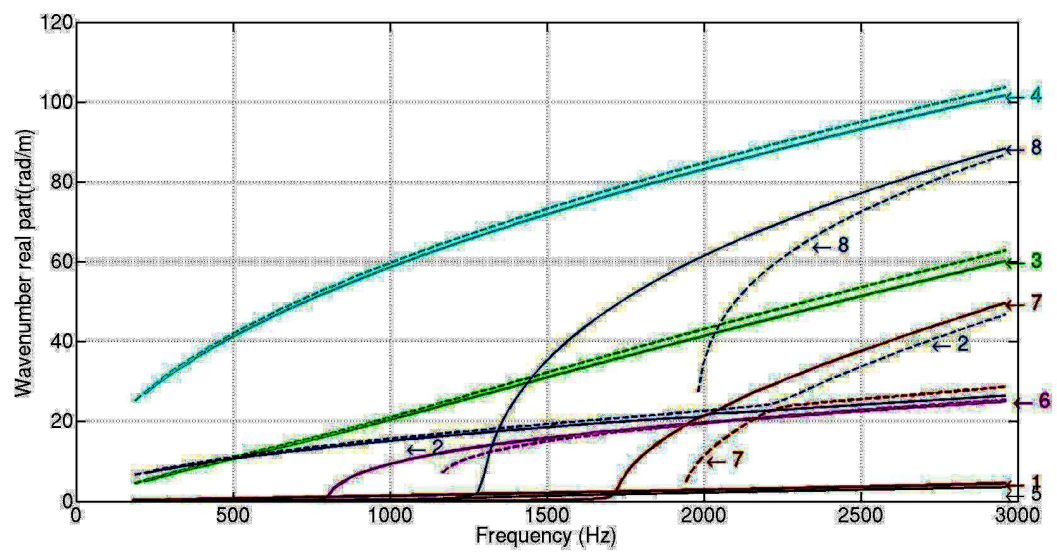

Figure 8: Dispersion curves for the global waveguide obtained using the first MWFE modeling, based on the reduced wave mode basis of each uncoupled layer. (solid lines)MWFE results (dashed lines)WFE results. 


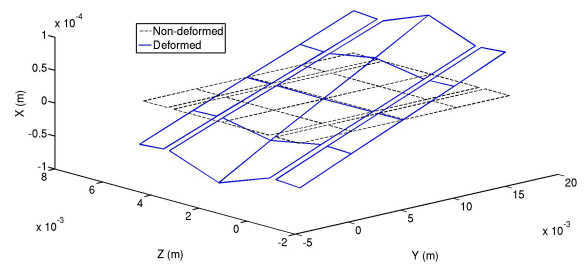

(a) Mode 2

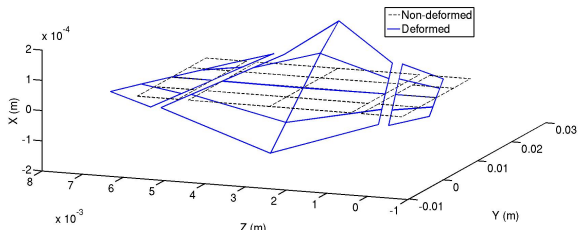

(b) Mode 7

Figure 9: Deformed modal shapes of (a)the $Y$-axis bending wave mode (Mode 2 ) and (b)the 2 nd $X$-axis torsional wave (Mode 7 ), at the frequency $f_{0}=$ $2000 \mathrm{~Hz}$. (solid line)Deformed mode shape (dashed line)undeformed crosssection. 


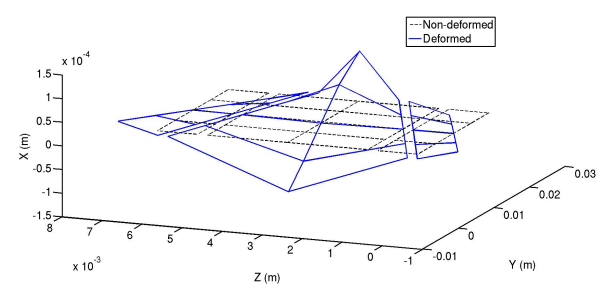

(a) Mode 2

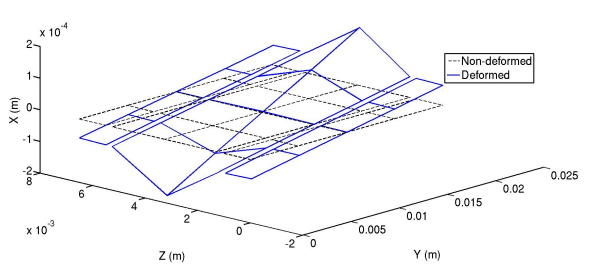

(b) Mode 7

Figure 10: Deformed modal shapes of (a)the $Y$-axis bending wave mode (Mode 2) and (b)the 2 nd $X$-axis torsional wave (Mode 7 ), at the frequency $f_{0}=2780 \mathrm{~Hz}$. (solid line)Deformed mode shape (dashed line)undeformed cross-section. 


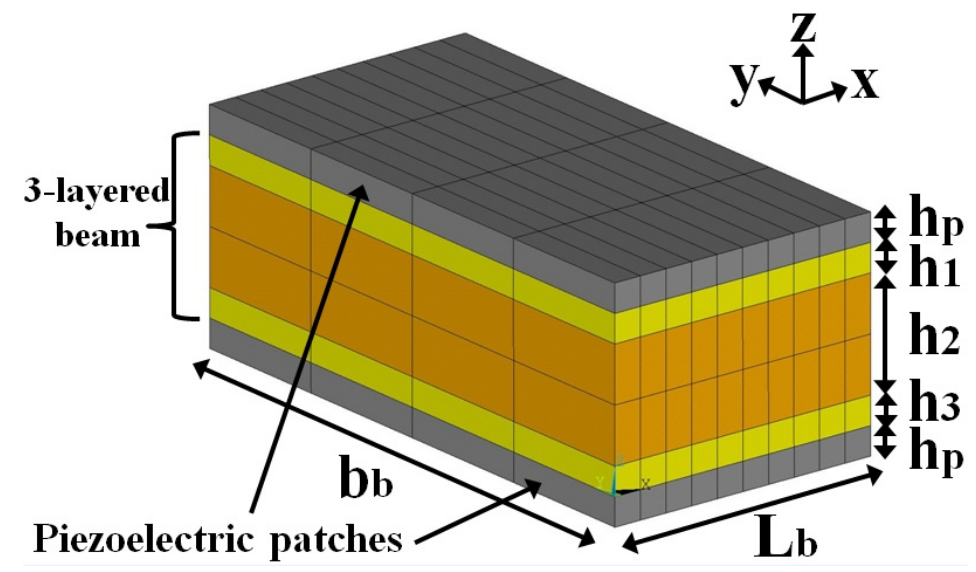

Figure 11: Finite element model of the coupling element in the three-layered system with 2 shunted piezoelectric patches. 


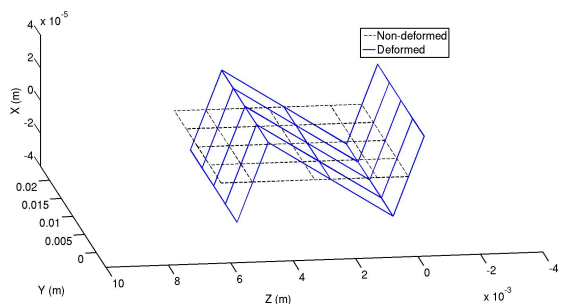

(a) WFE

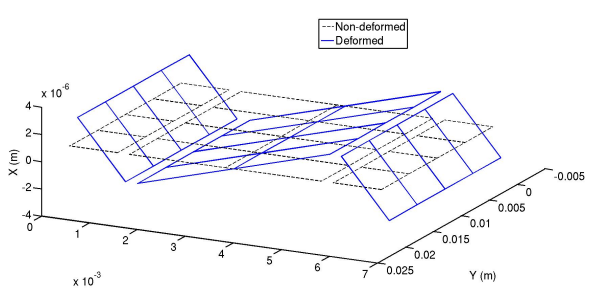

(b) MWFE

Figure 12: Deformed modal shapes of the $Z$-axis bending wave mode (Mode 4) issued from (a)WFE formulation (b)first MWFE formulation, at the frequency $f_{0}=380 \mathrm{~Hz}$. (solid line)Deformed mode shape (dashed line)undeformed cross-section. 

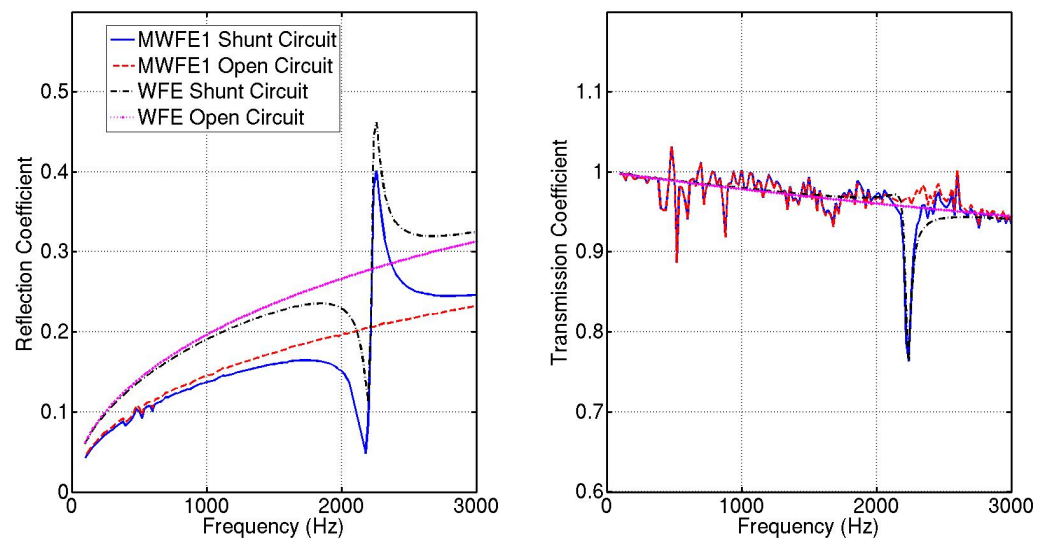

Figure 13: Reflection and transmission coefficients of the $Z$-axis bending wave mode using the first MWFE formulation and WFE method. 

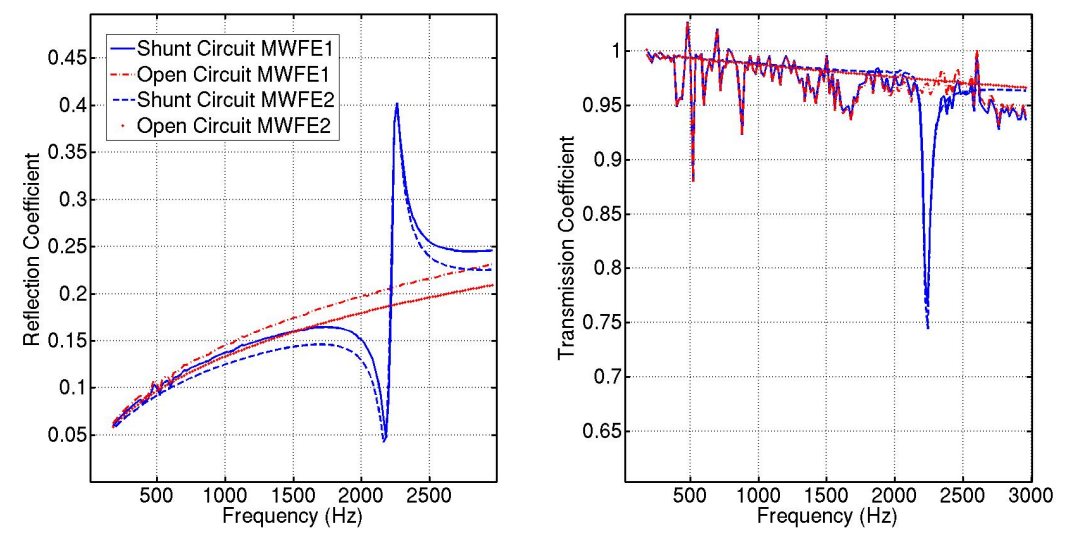

Figure 14: Reflection and transmission coefficients of the $Z$-axis bending wave mode using the first MWFE formulation and second MWFE method. 


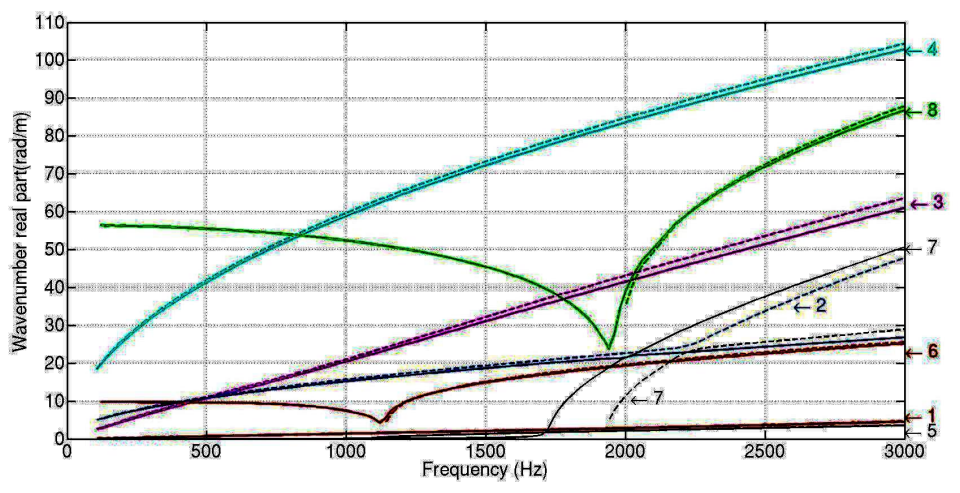

(a) original view

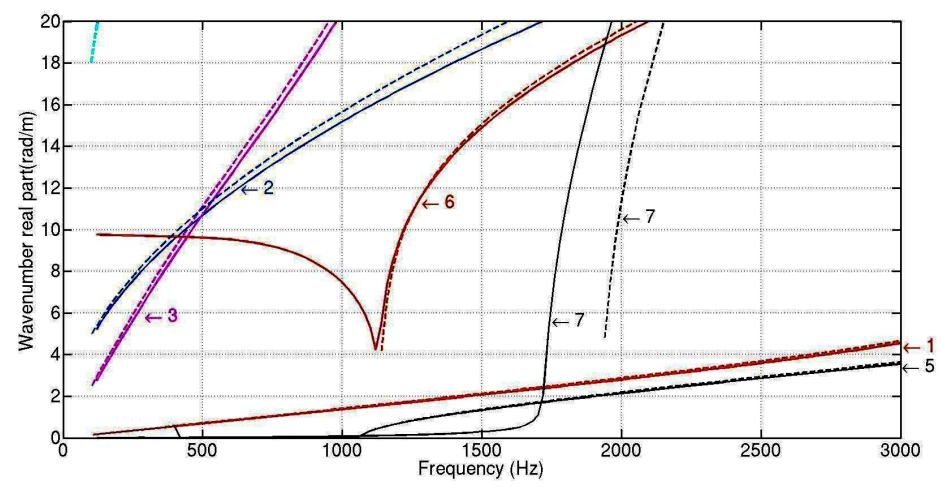

(b) zoom

Figure 15: Dispersion curves in the waveguide using the first MWFE formulation with different mode bases and WFE method. (solid lines)first MWFE results (dashed lines)WFE results. 


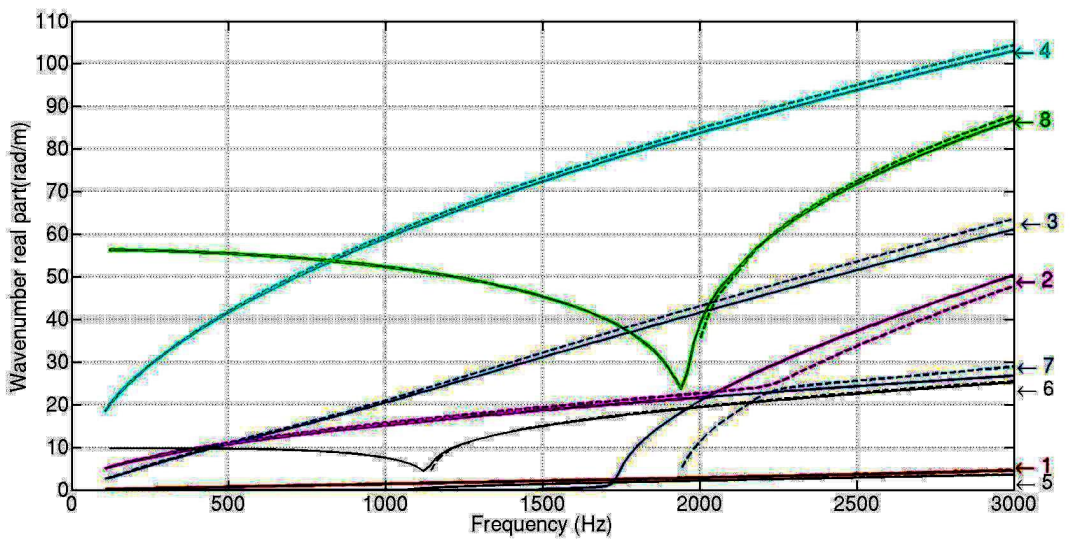

Figure 16: Dispersion curves in the waveguide using the second MWFE formulation with different mode bases and WFE method. (solid lines)second MWFE results (dashed lines)WFE results. 


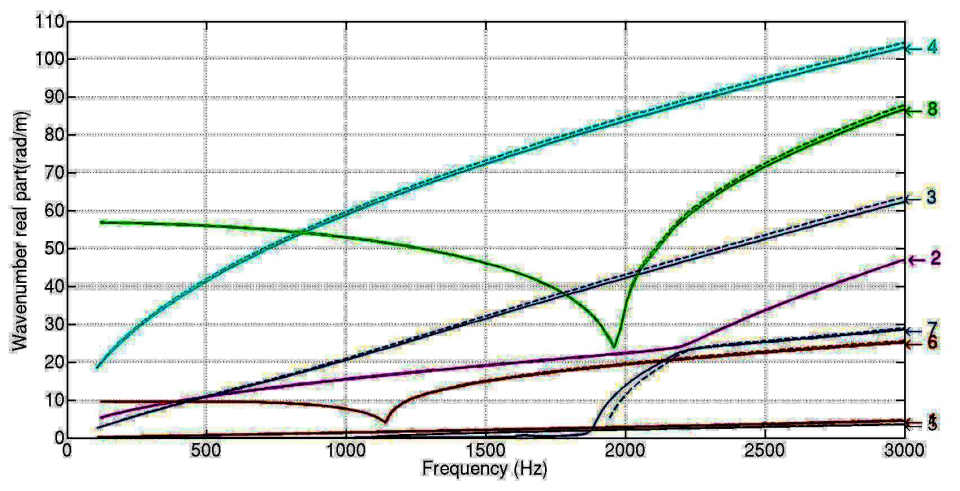

(a) original view

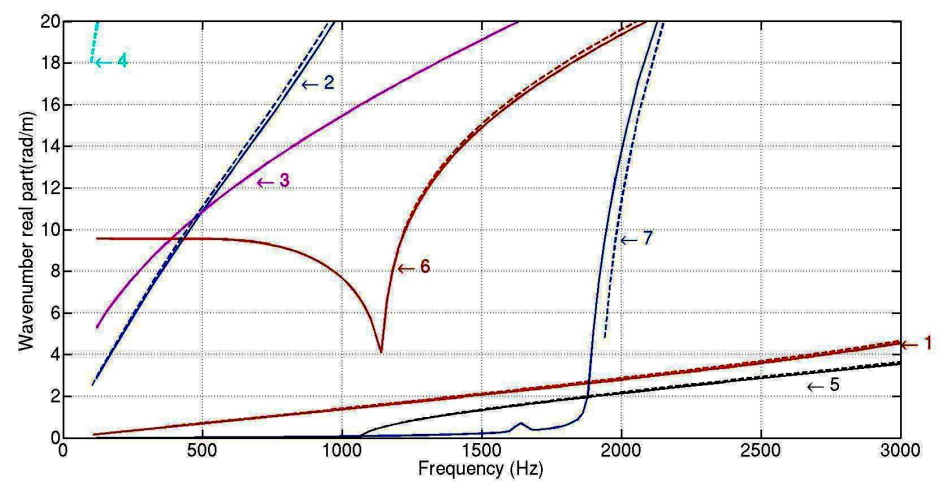

(b) zoom

Figure 17: Dispersion curves in the waveguide using the first MWFE formulation with different mode bases and WFE method. (solid lines)first MWFE results (dashed lines)WFE results. 


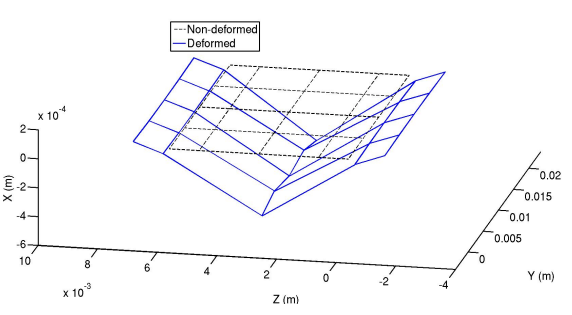

(a) WFE

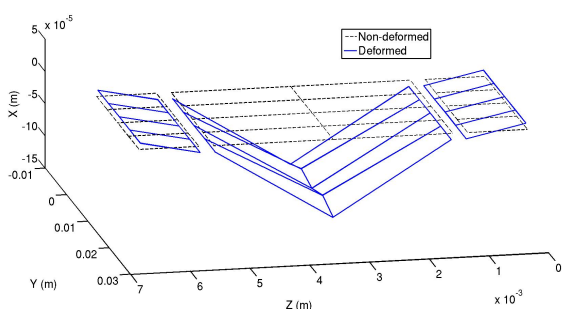

(b) MWFE

Figure 18: Deformed modal shapes of Mode 8 issued from (a)WFE formulation (b)second MWFE formulation, at the frequency $f_{0}=2780 \mathrm{~Hz}$. (solid line)Deformed mode shape (dashed line)undeformed cross-section. 

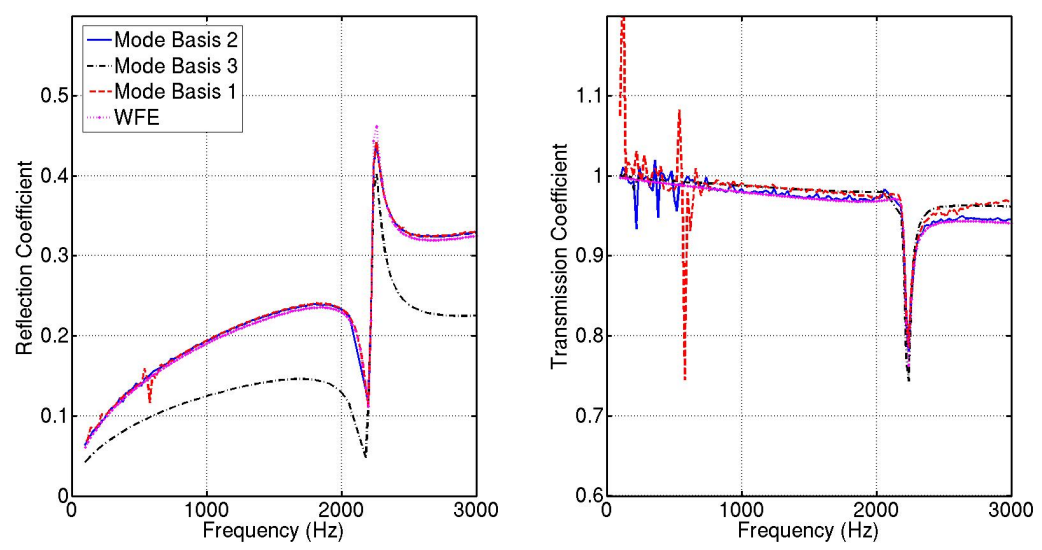

Figure 19: Reflection coefficients using the second MWFE formulation with different mode bases (1, 2 and 3) and WFE method. (solid line)Mode basis $2\left(m^{2}=30\right)$ (dashed line)Mode basis $1\left(m^{2}=40\right)$ (point-dashed line) Mode basis $3\left(m^{2}=45\right)$ (point markers)WFE results. $m^{1}=m^{3}=6$. 

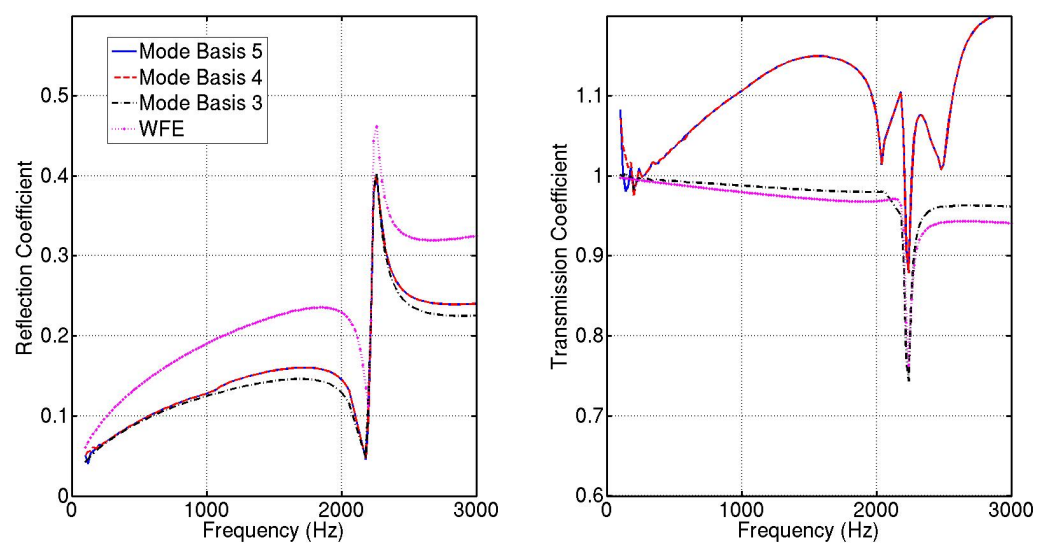

Figure 20: Reflection coefficients using the second MWFE formulation with different mode bases (3, 4 and 5) and WFE method. (solid line)Mode basis $5\left(m^{1}=m^{3}=4\right)$ (dashed line)Mode basis $4\left(m^{1}=m^{3}=5\right)$ (point-dashed line)Mode basis $3\left(m^{1}=m^{3}=6\right)$ (point markers)WFE results. $m^{2}=45$. 

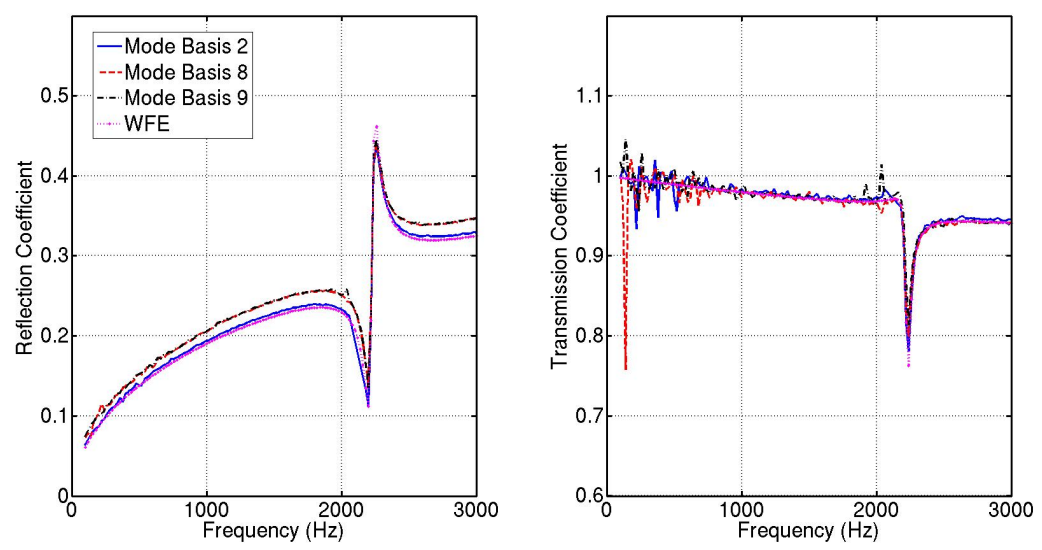

Figure 21: Reflection coefficients using the second MWFE formulation with different mode bases (2, 8 and 9) and WFE method. (solid line)Mode basis $2\left(m^{1}=m^{3}=6\right)$ (dashed line)Mode basis $8\left(m^{1}=m^{3}=5\right)$ (point-dashed line)Mode basis $9\left(m^{1}=m^{3}=4\right)$ (point markers)WFE results. $m^{2}=30$. 

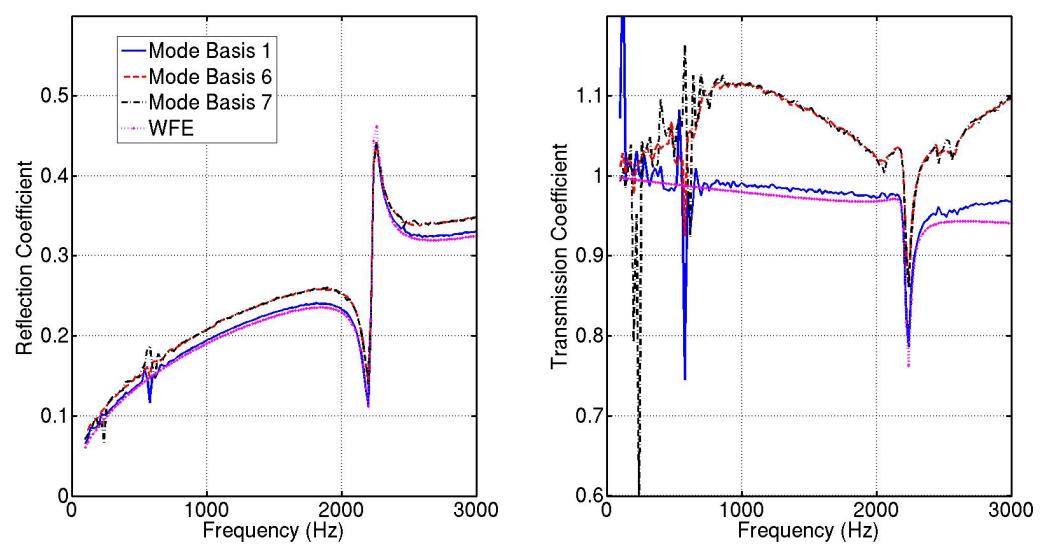

Figure 22: Reflection coefficients using the second MWFE formulation with different mode bases (1, 6 and 7) and WFE method. (solid line)Mode basis $1\left(m^{1}=m^{3}=6\right)$ (dashed line)Mode basis $6\left(m^{1}=m^{3}=5\right)$ (point-dashed line)Mode basis $7\left(m^{1}=m^{3}=4\right)$ (point markers)WFE results. $m^{2}=40$. 

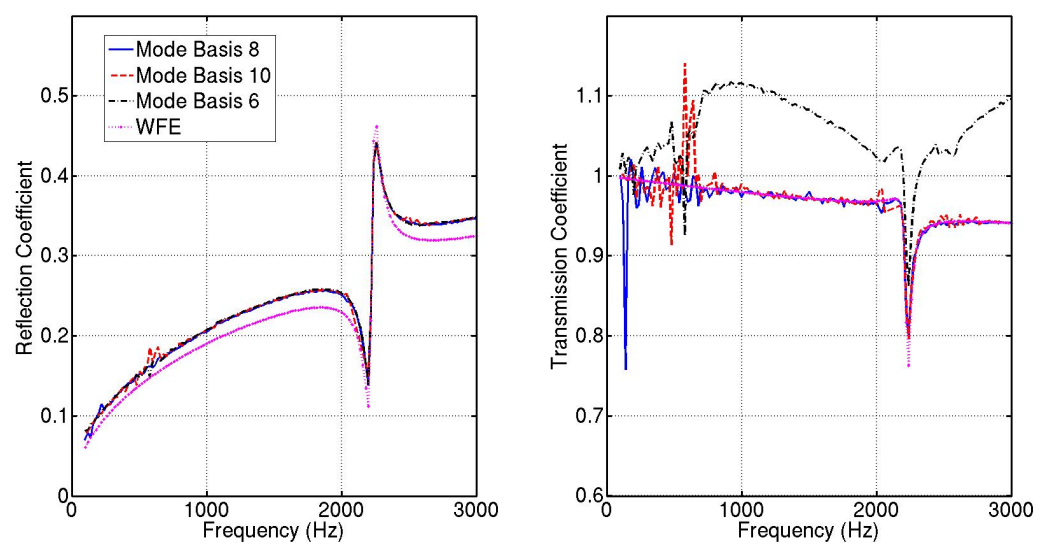

Figure 23: Reflection coefficients using the second MWFE formulation with different mode bases (6, 8 and 10) and WFE method. (solid line)Mode basis $8\left(m^{2}=30\right)$ (dashed line)Mode basis $10\left(m^{2}=36\right)$ (point-dashed line)Mode basis $6\left(m^{2}=40\right)$ (point markers)WFE results. $m^{1}=m^{3}=5$. 


\begin{tabular}{ccccccc}
\hline$d_{x}$ & $b_{b}$ & $h_{1}$ & $h_{2}$ & $h_{3}$ & $L_{b}$ & $h_{p}$ \\
\hline 0.001 & 0.02 & 0.001 & 0.004 & 0.001 & 0.01 & 0.001 \\
\hline
\end{tabular}

Table 1: Numeric values of the geometric parameters in the waveguide shown in Figures 5 and 11. The units of all the parameters are in meter $(m)$. 


\begin{tabular}{cccc}
\hline Mode Basis & $m^{1}$ & $m^{2}$ & $m^{3}$ \\
\hline 1 & 6 & 40 & 6 \\
\hline 2 & 6 & 30 & 6 \\
\hline 3 & 6 & 45 & 6 \\
\hline 4 & 5 & 45 & 5 \\
\hline 5 & 4 & 45 & 4 \\
\hline 6 & 5 & 40 & 5 \\
\hline 7 & 4 & 40 & 4 \\
\hline 8 & 5 & 30 & 5 \\
\hline 9 & 4 & 30 & 4 \\
\hline 10 & 5 & 36 & 5 \\
\hline
\end{tabular}

Table 2: Size of mode bases 